\title{
On the charge radius of the neutrino
}

\author{
J. Bernabéu, L. G. Cabral-Rosetti, J. Papavassiliou, and J. Vidal \\ Departamento de Física Teórica, Universidad de Valencia \\ E-46100 Burjassot (Valencia), Spain
}

\begin{abstract}
Using the pinch technique we construct at one-loop order a neutrino charge radius, which is finite, depends neither on the gauge-fixing parameter nor on the gauge-fixing scheme employed, and is process-independent. This definition stems solely from an effective proper photon-neutrino one-loop vertex, with no reference to box or self-energy contributions. The rôle of the $W W$ box in this construction is critically examined. In particular it is shown that the exclusion of the effective $W W$ box from the definition of the neutrino charge radius is not a matter of convention but is in fact dynamically realized when the target-fermions are right-handedly polarized. In this way we obtain a unique decomposition of effective self-energies, vertices, and boxes, which separately respect electroweak gauge invariance. We elaborate on the tree-level origin of the mechanism which enforces at one-loop level massive cancellations among the longitudinal momenta appearing in the Feynman diagrams, and in particular those associated with the non-abelian character of the theory. Various issues related to the known connection between the pinch technique and the Background Field Method are further clarified. Explicit closed expressions for the neutrino charge radius are reported.
\end{abstract}




\section{Introduction}

The neutrino electromagnetic form-factor and the corresponding neutrino charge radius (NCR) are interesting physical quantities, mainly because their non-vanishing appreciable values could be attributed to physics beyond the Standard Model (SM). Within the SM, one expects that an effective photon-neutrino interaction will be generated through one-loop radiative corrections, giving rise to a non-zero, albeit small NCR. However, the direct calculation of this quantity has been faced with serious complications [1-4] which, in turn, can be traced back to the fact that in non-Abelian gauge theories off-shell Green's functions depend in general explicitly on the gaugefixing parameter (GFP). Therefore the definition of quantities familiar from scalar theories of QED, such as effective charges and form-factors, is in general problematic. In the case of the NCR, various attempts to define its SM value through the one-loop $\gamma \nu \nu$ vertex calculated in the renormalizable $\left(R_{\xi}\right)$ gauges reveal that the corresponding electromagnetic form-factor $F_{1}\left(q^{2}, \xi\right)$ depends explicitly on the GFP in a prohibiting way. In particular, even though in the static limit of zero momentum transfer, $q^{2} \rightarrow 0$, the form-factor $F_{1}\left(q^{2}, \xi\right)$ becomes independent of $\xi$, its first derivative with respect to $q^{2}$, which corresponds to the classic definition of the NCR, namely $\left\langle r_{\nu}^{2}\right\rangle=-\left.6 \frac{\partial F_{1}\left(q^{2}, \xi\right)}{\partial q^{2}}\right|_{q^{2}=0}$, continues to depend on it.

The problems associated with the definition of the NCR within the SM and beyond have been examined in detail in a series of papers, and various proposals have been put forth on how to bypass them [5-16]. The main idea behind these works has been to exploit judiciously the GFP-independence of the entire physical amplitude, and eventually define a piece of it which is kinematically akin to an electromagnetic form factor, and is in addition endowed with a set of important physical properties. In this context the rôle of the box diagrams containing two $W$ 's has presented a long-standing puzzle. It was recognized in [17] that this box contains in general crucial GFP-dependent pieces, which, when included to the NCR definition render the answer GFP-independent. Thus, whereas in this construction the ZZ boxes have been naturally discarded, since they do not participate non-trivially in the gauge cancellations of the NCR, the $W W$ box is instead included in the definition, because it is important for the gauge cancellations. The disadvantage of including the box however is that it introduces a process-dependence, since it depends explicitly on the quantum numbers of the target fermions; this fact in turn would prevent the NCR from being regarded as a target-independent property, intrinsic to the neutrinophoton interaction. Even though the process-dependent contributions may become numerically subleading when studying the low-energy static limit, where the NCR has a direct electromagnetic analogue, from the theoretical point of view one would like to be able to extend its notion into that of an effective form-factor, which would be GFP- and process-independent for arbitrary values of the momentum transfer.

The way out of this dilemma has been presented in [18], in the context of the pinch technique (PT) [19-22]: one can construct a genuine, target-independent form-factor endowed with the crucial properties of gauge-independence, gauge-invariance and finiteness, for arbitrary values of the momentum transfer. The essential ingredient for accomplishing this was the realization that, when the target fermions involved are considered to be massless, all GFP-dependent contributions stemming from box and vertex-like Feynman diagrams are effectively propagator-like, i.e. have no dependence on the kinematic properties or quantum numbers of the initial and final states, as boxes and vertices normally do. The precise mechanism which allows the identification and extraction of these propagator-like contributions from boxes and vertices relies on the systematic 
exploitation of fundamental tree-level Ward identities (WI). At tree-level these WI enforce crucial cancellations between $s$-and $t$-channel contributions in the $W$-pair production process $[23,24]$. Their one-loop descendant is a set of vast cancellations involving contributions originating from seemingly dissimilar Feynman graphs. Thus, all gauge dependence stemming from vertices and boxes cancels precisely against the gauge-dependence stemming from the conventional one-loop self-energies. As a result, the remaining GFP-independent structures retain their initial kinematic identity; in particular one can speak in terms of GFP-independent effective boxes, vertices, and self-energies. Thus, once the GFP-dependent pieces have been extracted from the box, the remaining GFP-independent "pure" box should not be considered as a part of the resulting form-factor, which should be entirely determined from the "pure" GFP-independent set of vertex graphs. Notice that, in the original proposal, the gamma Z self-energy constituted part of the form-factor [18]; whereas this is clearly a process-independent contribution it became clear recently [24] that the $\gamma Z$ self-energy should not be part of the form-factor either, since its interpretation as part of the electro-weak effective charge is physically more appealing.

Ii should be emphasized that the aforementioned rearrangement of the S-matrix into individually GFP-independent effective boxes, vertices, and self-energies occurs automatically, at least up to two-loops [25], if one adheres to the well-defined set of PT rules, which may be summarized as follows: Identify all longitudinal momenta, i.e. all momenta which can trigger the aforementioned elementary Ward identities, and track down the resulting algebraically realized cancellations. One, but not the only, consequence of the above procedure is that all dependence on the GFP is effectively self-energy-like, and finally vanishes since the S-matrix is guaranteed to be GFP-independent. Notice however that longitudinal momenta do not only originate from the tree-level propagators appearing in the graphs but also from the appropriate decomposition of the elementary tri-linear gauge-boson vertices [26]. This decomposition is characteristic of the PT and has far-reaching consequences; in particular, the resulting effective one-and two-loop Green's functions satisfy QED-like WI, instead of the usual complicated Slavnov-Taylor identities. In addition, they coincide, at least up to two-loops [27,25], with the results one would obtain if one were to calculate the Green's functions in the Background Field Method (BFM) [28-31] at the special value of the (quantum) GFP $\xi_{Q}^{W}=1$. It is important to appreciate that the aforementioned vertex decomposition has to be carried out even if all gauge dependences have explicitly cancelled; conversely, it can be carried out at the beginning of the calculation, before any of the GFP cancellations have been implemented, i.e the order of triggering the WI and identifying the various propagator-like pieces is immaterial, as it should.

Even though the correct interpretation of the correspondence between the PT and the BFM at $\xi_{Q}^{W}=1$ has been discussed in the literature [23,24,32], it has often been misinterpreted as constituting an arbitrary choice rather than a result singled out in a unique way by the full exploitation of the underlying Becchi-Ruet-Stora (BRS) symmetries [33], intrinsic to the S-matrix (or any ostensibly gauge-invariant quantity such as a Wilson loop, for example). In addition, the proposal to exclude the pure box from the NCR on the grounds of the process-dependence that it induces has not been implemented when imposing experimental bounds on the NCR [34-37]. The confusion surrounding the rôle of the box may be amplified by arguments supporting the plausible scenario according to which parts of the "pure" box, which could be obtained after the integration over virtual momenta, may be incorporated into the definition of the NCR without violating any of the physical principles in any obvious way. (Notice that the PT rearrangement of the amplitude takes place before any integrations over virtual momenta have been carried out). 
In this paper we demonstrate that the exclusion of the "pure" or "effective" $W W$ box from the definition of the NCR is not a matter of convention but is in fact dynamically realized in a particular physical process, namely when the target-fermions are right-handedly polarized, simply because in that case the $W W$ box is not there to begin with. In particular we demonstrate explicitly that the PT construction goes through unmodified in the absence of the box, because the cancellations previously enforced by parts of the box are now enforced by the modification of the couplings of the photon and the $Z$ to right-handed fermions. In particular, at tree-level the $\gamma f_{R} \bar{f}_{R}$ and $Z f_{R} \bar{f}_{R}$ couplings allow for a cancellation involving the two $s$-channels graphs, one mediated by an off-shell photon, and one by an off-shell $Z$. This cancellation replaces the usual $s$-channel - $t$-channel cancellation occuring in the case of unpolarized fermions [23,24], since now there is no $t$-channel graphs involving two outgoing $W$. The aforementioned tree-level cancellation is in fact responsible for the one-loop cancellations we will demonstrate in detail. The analysis carried out settles conclusively the question of whether after the gauge cancellations have been implemented the pure box (or some arbitrarily chosen parts of it) should be part of the definition of the form factor giving rise to the NCR. In addition, the explicit demonstration of the gauge cancellations in two different gauge-fixing schemes $\left(R_{\xi}\right.$ and BFM) shows the general validity and self-consistency of the entire procedure, and, as a by-product, it makes abundantly clear the fact that (in the latter case) at no point the choice $\xi_{Q}^{W}=1$ has been a-priori implemented.

At the end of this procedure, we obtain a neutrino electromagnetic form-factor which (a) is independent of the GFP; (b) couples electromagnetically to the target; (c) it is process (target)independent and can therefore be considered as an intrinsic property of the neutrino; (d) the effective one-loop $\gamma \nu \bar{\nu}$ proper vertex from which it originates satisfies a QED-like WI; (e) it holds for arbitrary values (time-like and space-like ) of the four-momentum transfer (f) since the selfenergies do not enter into its definition, the resulting NCR does not depend on the quark sector of the theory.

The paper is organized as follows: In section 2 we present a general discussion on the PT. In Section 3 we demonstrate explicitly the gauge cancellations in the context of the $R_{\xi}$ gauges, for unpolarized target fermions; here the $W W$ box appears and its participation in the aforementioned cancellations is crucial. In section 4 we demonstrate how the crucial cancellations are enforced in the case of right-handedly polarized target fermions, i.e. when the $W W$ box is actually absent. In section 5 we present in detail how the above cancellations are enforced in the context of the BFM. In section 6 we show how the final rearrangement of the physical amplitude according to the general PT rules singles out the final gauge-invariant answer for the individual effective self-energies, vertices, and boxes; the latter quantities coincide with their conventionally defined counterparts calculated in the Feynman gauge of the BFM. In section 7 we present explicit results

for the NCR extracted from the effective photon-neutrino vertex, and the slope $\left(\right.$ at $q^{2}=0$ ) of the effective charge defined through the PT $\gamma Z$ self-energy. Finally, in section 8 we present our conclusions.

\section{General discussion}

In this section we outline the field-theoretical mechanism which enforces the massive gauge cancellations and allows for the construction of GFP-independent and gauge-invariant boxes, vertices, and self-energies. The NCR will then be defined from the one-loop $\gamma \nu \nu$ vertex so constructed. 
The longitudinal momenta appearing in the $S$-matrix element of $F \bar{F} \rightarrow \nu \bar{\nu}$ originate from the tree-level gauge-boson propagators and tri-linear gauge-boson vertices appearing inside loops. In particular, in the $R_{\xi}$-scheme the gauge-boson propagators have the general form

$$
\Delta_{V}^{\mu \nu}(k)=\left[g^{\mu \nu}-\frac{\left(1-\xi_{V}\right) k^{\mu} k^{\nu}}{k^{2}-\xi_{V} M_{V}^{2}}\right] D_{V}(k)
$$

with

$$
D_{V}(k)=\left(k^{2}-M_{V}^{2}\right)^{-1}
$$

where $V=W, N$ with $N=Z, \gamma$ and $M_{\gamma}^{2}=0 ; k$ denotes the virtual four-momentum circulating in the loop. Clearly, in the case of $\Delta_{V}^{\mu \nu}(k)$ the longitudinal momenta are those proportional to $\left(1-\xi_{V}\right)$. The longitudinal terms arising from the tri-linear vertex may be identified by splitting $\Gamma_{\alpha \mu \nu}(q, k,-k-q)$ appearing inside the one-loop diagrams (see Fig.1a and Fig.2 for the definition of the momenta entering into the vertex) into two parts ( $q$ denotes the physical four-momentum entering into the vertex)[26]:

$$
\begin{aligned}
\Gamma_{\alpha \mu \nu}(q, k,-k-q) & =(q-k)_{\nu} g_{\alpha \mu}+(2 k+q)_{\alpha} g_{\mu \nu}-(2 q+k)_{\mu} g_{\alpha \nu} \\
& =\Gamma_{\alpha \mu \nu}^{F}+\Gamma_{\alpha \mu \nu}^{P},
\end{aligned}
$$

with

$$
\begin{aligned}
& \Gamma_{\alpha \mu \nu}^{F}=(2 k+q)_{\alpha} g_{\mu \nu}+2 q_{\nu} g_{\alpha \mu}-2 q_{\mu} g_{\alpha \nu}, \\
& \Gamma_{\alpha \mu \nu}^{P}=-(k+q)_{\nu} g_{\alpha \mu}-k_{\mu} g_{\alpha \nu} .
\end{aligned}
$$

The first term in $\Gamma_{\alpha \mu \nu}^{F}$ is a convective vertex describing the coupling of a vector boson to a scalar field, whereas the other two terms originate from spin or magnetic moment. The above decomposition assigns a special rôle to the $q$-leg, and allows $\Gamma_{\alpha \mu \nu}^{F}$ to satisfy the Ward identity

$$
q^{\alpha} \Gamma_{\alpha \mu \nu}^{F}=(k+q)^{2} g_{\mu \nu}-k^{2} g_{\mu \nu},
$$

All aforementioned longitudinal momenta originating from $\Delta_{V}^{\mu \nu}(k)$ and $\Gamma_{\alpha \mu \nu}^{P}$ trigger the following WI when contracted with the appropriate $\gamma$ matrix appearing in the various elementary vertices :

$$
\begin{aligned}
\not k P_{L} & =(\not k+\not \not) P_{L}-P_{R} \not p, \\
& =S_{F^{\prime}}^{-1}(\not k+\not \not) P_{L}-P_{R} S_{F}^{-1}(\not p)+m_{F^{\prime}} P_{L}-m_{F} P_{R}
\end{aligned}
$$

where $P_{R(L)}=\left[1+(-) \gamma_{5}\right] / 2$ is the chirality projection operator and $S_{F}$ is the tree-level propagator of the fermion $F$; $F^{\prime}$ is the isodoublet-partner of the external fermion $F$. The result of this contraction is that the term in Eq.(2.6) proportional to $S_{F^{\prime}}^{-1}$, i.e the inverse of the internal fermion propagator gives rise to a self-energy-like term, whose coupling to the external fermions is proportional to the vertex

$$
\Gamma_{W F \bar{F}}^{\mu}=-i\left(\frac{g_{w}}{2}\right) \gamma_{\mu} P_{L} .
$$

The effective vertex $\Gamma_{W F \bar{F}}^{\mu}$ should not be confused with the usual $W F F^{\prime}$ elementary vertex involving two fermions $F$ and $F^{\prime}$ of different charge and isospin. The vertex $\Gamma_{W F \bar{F}}^{\mu}$ can be written as a linear combination of the two tree-level vertices $\Gamma_{\gamma F \bar{F}}^{\mu}$ and $\Gamma_{Z F \bar{F}}^{\mu}$ given by

$$
\begin{aligned}
\Gamma_{\gamma F \bar{F}}^{\mu} & =-i g_{w} s_{w} Q_{F} \gamma^{\mu} \\
\Gamma_{Z F \bar{F}}^{\mu} & =-i\left(\frac{g_{w}}{c_{w}}\right) \gamma^{\mu}\left[\left(s_{w}^{2} Q_{F}-T_{z}^{F}\right) P_{L}+s_{w}^{2} Q_{F} P_{R}\right]
\end{aligned}
$$


as follows:

$$
\Gamma_{W F \bar{F}}^{\mu}=\left(\frac{s_{w}}{2 T_{z}^{F}}\right) \Gamma_{\gamma F \bar{F}}^{\mu}-\left(\frac{c_{w}}{2 T_{z}^{F}}\right) \Gamma_{Z F \bar{F}}^{\mu}
$$

In the above formulas $Q_{F}$ is the electric charge of the fermion $F$, and $T_{z}^{F}$ its $z$-component of the weak isospin, with $c_{w}=\sqrt{1-s_{w}^{2}}=M_{W} / M_{Z}, e=g_{w} s_{w}$,

The identity presented in Eq.(2.9) allows to combine the propagator-like parts emerging from box-diagrams and vertex-diagrams after the application of the WI in Eq.(2.6) with the conventional self-energy graphs $\Pi_{\mu \nu}^{\gamma Z}$ and $\Pi_{\mu \nu}^{Z Z}$ (Fig. 1), by judiciously multiplying by $D_{N}(q) D_{N}^{-1}(q)$, as shown in Fig. 2 - Fig. 4 . In fact, in order to see exactly against which parts of the self-energies the aforementioned terms will cancel, one must notice [38] that every time there is longitudinal momentum in a vertex or a box diagram triggering the WI in Eq.(2.6), the same longitudinal momentum inside a self-energy diagram will be contracted with one of the elementary tri-linear vertices, triggering a WI of the form

$$
\begin{aligned}
(k+q)^{\nu} \Gamma_{\alpha \mu \nu}(q, k,-k-q) & =\left(q^{2} g_{\alpha \mu}-q_{\alpha} q_{\mu}\right)-\left(k^{2} g_{\alpha \mu}-k_{\alpha} k_{\mu}\right) \\
& =\left(D_{N}^{-1}(q)-D_{W}^{-1}(k)+\left(M_{N}^{2}-M_{W}^{2}\right)\right) g_{\alpha \mu}+k_{\alpha} k_{\mu}
\end{aligned}
$$

The terms proportional to $D_{N}^{-1}(q)$ appearing in Eq.(2.10) are precisely those which will cancel against the aforementioned propagator-like pieces coming from vertices and boxes. It is clear that the exploitation of the elementary WI of Eq.(2.6) and Eq.(2.10) enable the communication between Feynman diagrams which at first seem to be kinematically distinct.

It turns out that the one-loop cancellations described above descend in fact from a fundamental tree-level cancellation between $s$ - and $t$ - channel diagrams, the latter being a consequence of the underlying BRS symmetry [23,24]. For the remainder of this paper we will restrict our attention to the case where the fermions $F$ are leptons, to be denoted by $f$; in that case $T_{z}^{f}=-\frac{1}{2}$ and for the neutrino $T_{z}^{\nu_{e}}=\frac{1}{2}$ and so the identity becomes

$$
\begin{aligned}
& \Gamma_{W f \bar{f}}^{\mu}=-s_{w} \Gamma_{\gamma f \bar{f}}^{\mu}+c_{w} \Gamma_{Z f \bar{f}}^{\mu} \\
& \Gamma_{W \nu \bar{\nu}}^{\mu}=-c_{w} \Gamma_{Z \nu \bar{\nu}}^{\mu}
\end{aligned}
$$

To see how the aforementioned tree-level cancellation is enforced, consider the process $f \bar{f} \rightarrow$ $W^{+} W^{-}$, whose tree-level amplitude will be denoted by $\mathcal{T}_{\mu \nu}$. Defining

$$
\mathcal{V}_{V f \bar{f}}^{\mu}=\bar{v}_{f}\left(p_{2}\right) \Gamma_{V f \bar{f}}^{\mu} u_{f}\left(p_{1}\right)
$$

we have that $\mathcal{T}_{\mu \nu}$ is given by two $s$-channel and one $t$-channel graphs,

$$
\mathcal{T}_{\mu \nu}=\mathcal{T}_{s \mu \nu}^{(\gamma)}+\mathcal{T}_{s \mu \nu}^{(z)}+\mathcal{T}_{t \mu \nu}
$$

where

$$
\begin{aligned}
& \mathcal{T}_{s \mu \nu}^{(\gamma)}=\mathcal{V}_{\gamma f \bar{f}}^{\alpha} D_{\gamma}(q) \Gamma_{\alpha \mu \nu}^{\gamma W W}\left(q, k_{1}, k_{2}\right) \\
& \mathcal{T}_{s \mu \nu}^{(z)}=\mathcal{V}_{Z f \bar{f}}^{\alpha} D_{z}(q) \Gamma_{\alpha \mu \nu}^{Z W W}\left(q, k_{1}, k_{2}\right) \\
& \mathcal{T}_{\mu \nu}^{(t)}=-\frac{g_{w}^{2}}{2} \bar{v}_{f}\left(p_{2}\right) \gamma^{\mu} P_{L} S_{f^{\prime}}\left(\not p_{1}-\not k_{1}\right) \gamma^{\nu} P_{L} u_{f}\left(p_{1}\right)
\end{aligned}
$$


with

$$
\begin{aligned}
& \Gamma_{\alpha \mu \nu}^{\gamma W W}\left(q, k_{1}, k_{2}\right)=-g_{w} s_{w} \Gamma_{\alpha \mu \nu}\left(q, k_{1}, k_{2}\right) \\
& \Gamma_{\alpha \mu \nu}^{Z W W}\left(q, k_{1}, k_{2}\right)=g_{w} c_{w} \Gamma_{\alpha \mu \nu}\left(q, k_{1}, k_{2}\right)
\end{aligned}
$$

Here, $s=q^{2}=\left(p_{1}+p_{2}\right)^{2}=\left(k_{1}+k_{2}\right)^{2}$ is the c.m. energy squared.

Let us now consider the case where $\mathcal{T}_{\mu \nu}$ is contracted by a longitudinal momentum $k_{1}^{\mu}$ or $k_{2}^{\nu}$. Then, the WI of Eq.(2.10) will operate at the two $s$-channel graphs, whereas that of Eq.(2.6) at the $t$-channel graph (Fig. 5), yielding

$$
\begin{aligned}
k_{1 \mu} \mathcal{T}_{s(\gamma)}^{\mu \nu} & =-s_{w} \mathcal{V}_{\gamma f \bar{f}}^{\nu}+S^{(\gamma) \nu} \\
k_{1 \mu} \mathcal{T}_{s(z)}^{\mu \nu} & =c_{w} \mathcal{V}_{Z f \bar{f}}^{\nu}+S^{(z) \nu} \\
k_{1 \mu} \mathcal{T}_{(t)}^{\mu \nu} & =\mathcal{V}_{W f \bar{f}}^{\nu}
\end{aligned}
$$

with

$$
\begin{aligned}
S_{\nu}^{(z)} & =\mathcal{V}_{Z f \bar{f}}^{\alpha} D_{Z}(q) c_{w}\left(\left(M_{Z}^{2}-M_{W}^{2}\right) g_{\alpha \nu}+k_{2 \alpha} k_{2 \nu}\right) \\
S_{\nu}^{(\gamma)} & =\mathcal{V}_{\gamma f \bar{f}}^{\alpha} D_{Z}(q) s_{w}\left(M_{W}^{2} g_{\alpha \nu}-k_{2 \alpha} k_{2 \nu}\right)
\end{aligned}
$$

Adding by parts both sides of Eq.(2.16) we see that a major cancellation takes place; in particular the pieces containing the vertices $\mathcal{V}_{V f \bar{f}}^{\alpha}$ cancel by virtue of the first identity in Eq.(2.11), and one is left on the RHS with purely s-channel contribution, i.e

$$
k_{1}^{\mu} \mathcal{T}_{\mu \nu}=S_{\nu}^{(z)}+S_{\nu}^{(\gamma)}
$$

It is important to recognize that the cancellation described above goes through even when the initial fermions are right-handedly polarized, although in that case there is no $t$-channel graph (Fig. 6). In fact, the elementary vertices given in Eq.(2.8), together with the corresponding $\mathcal{V}_{\gamma f \bar{f}}^{\mu}$ and $\mathcal{V}_{Z f \bar{f}}^{\mu}$ are appropriately modified. In particular,

$$
\begin{aligned}
\Gamma_{\gamma_{f_{R} \bar{f}_{R}}}^{\mu} & =-i g_{w} s_{w} Q_{f} \gamma^{\mu} \\
\Gamma_{Z f_{R} \bar{f}_{R}}^{\mu} & =-i\left(\frac{g_{w}}{c_{w}}\right) s_{w}^{2} Q_{f} \gamma^{\mu}
\end{aligned}
$$

and

$$
\mathcal{V}_{Z f_{R} \bar{f}_{R}}^{\mu}=\left(\frac{s_{w}}{c_{w}}\right) \mathcal{V}_{\gamma_{f_{R} \bar{f}_{R}}^{\mu}}
$$

Clearly, in that case, from Eq. (2.11) follows immediately that

$$
\mathcal{V}_{W f_{R} \bar{f}_{R}}^{\mu}=0 .
$$

and Eq. (2.18) is still satisfied. 


\section{The unpolarized case in the $R_{\xi}$ gauge}

In this section we will demonstrate explicitly how the cancellation of the GFP proceeds in the case when the theory is quantized in the framework of the renormalizable $R_{\xi}$ gauge. Of course, based on general field-theoretical principles, one knows in advance that the entire amplitude will be GFP-independent. What we will show in this section is that in fact this cancellation goes through without having to carry out any of the integrations over virtual loop momenta. Indeed, even though individual graphs conspire non-trivially in order for the cancellations to take place, the way this happens kinematically is very distinct. In particular, all GFP-dependent parts are self-energy like; their identification and extraction from box and vertex diagrams is implemented exclusively through the use of elementary WI. Therefore, the remaining GFP-independent parts of each diagram retain their original kinematic identity, i.e. one may still identify box-, vertex-, and self-energy-like sub-amplitudes [39].

We will focus on the case where the target fermions are charged leptons, which we denote by $f$; the generalization to the case where the target fermions are quarks is straightforward. In what follows we will set $\lambda_{W} \equiv 1-\xi_{W}$, and we will suppress a factor $g_{w}^{2} \int \frac{d^{n} k}{(2 \pi)^{n}}$, where $n=4-\epsilon$ is the dimension of space-time in dimensional regularization. In addition we define

$$
\begin{aligned}
& I_{0}=\left[\left(k^{2}-M_{W}^{2}\right)\left((k+q)^{2}-M_{W}^{2}\right)\right]^{-1} \\
& I_{1}=\left[\left(k^{2}-\xi_{W} M_{W}^{2}\right)\left(k^{2}-M_{W}^{2}\right)\right]^{-1} \\
& I_{2}=\left[\left(k^{2}-\xi_{W} M_{W}^{2}\right)\left((k+q)^{2}-\xi_{W} M_{W}^{2}\right)\right]^{-1} \\
& I_{3}=\left[\left(k^{2}-\xi_{W} M_{W}^{2}\right)\left(k^{2}-M_{W}^{2}\right)\left((k+q)^{2}-M_{W}^{2}\right)\right]^{-1} \\
& I_{4}=\left[\left(k^{2}-\xi_{W} M_{W}^{2}\right)\left((k+q)^{2}-\xi_{W} M_{W}^{2}\right)\left(k^{2}-M_{W}^{2}\right)\left((k+q)^{2}-M_{W}^{2}\right)\right]^{-1} \\
& I_{5}=\left(k^{2}-\xi_{W} M_{W}^{2}\right)
\end{aligned}
$$

and we will employ the algebraic identity

$$
\frac{1}{k^{2}-\xi_{W} M_{W}^{2}}=\frac{1}{k^{2}-M_{W}^{2}}-\frac{\left(1-\xi_{W}\right) M_{W}^{2}}{\left(k^{2}-M_{W}^{2}\right)\left(k^{2}-\xi_{W} M_{W}^{2}\right)}
$$

in order to rearrange various expressions. In addition, we will drop terms proportional to $q_{\mu}$ or $q_{\nu}$ because we assume conserved external currents (mass-less fermions)

Turning to individual diagrams, we first consider the box graphs shown in Fig. 7. We have:

$$
\begin{aligned}
(n) & =(n)_{\xi_{W}=1}+\mathcal{V}_{W f \bar{f}}^{\mu}\left(\lambda_{W}^{2} I_{4} k_{\mu} k_{\nu}-2 \lambda_{W} I_{3} g_{\mu \nu}\right) \mathcal{V}_{W \nu \bar{\nu}}^{\nu} \\
& =(n)_{\xi_{W}=1}+(n)_{\gamma Z}+(n)_{Z Z}
\end{aligned}
$$


with

$$
\begin{aligned}
& (n)_{\gamma Z}=\mathcal{V}_{\gamma f \bar{f}}^{\mu} D_{\gamma}(q)\left[s_{w} c_{w} D_{\gamma}^{-1}(q)\left(\lambda_{W}^{2} I_{4} k_{\mu} k_{\nu}-2 \lambda_{W} I_{3} g_{\mu \nu}\right) D_{Z}^{-1}(q)\right] D_{Z}(q) \mathcal{V}_{Z \nu \bar{\nu}}^{\nu} \\
& (n)_{Z Z}=-\mathcal{V}_{Z f \bar{f}}^{\mu} D_{Z}(q)\left[c_{w}^{2} D_{Z}^{-1}(q)\left(\lambda_{W}^{2} I_{4} k_{\mu} k_{\nu}-2 \lambda_{W} I_{3} g_{\mu \nu}\right) D_{Z}^{-1}(q)\right] D_{Z}(q) \mathcal{V}_{Z \nu \bar{\nu}}^{\nu}
\end{aligned}
$$

Notice that $(n)_{\gamma z}$ and $(n)_{z z}$ are propagator-like quantities

For the vertex graphs $(o),(p)$, and $(q)$ we have (there is a relative minus sign from the difference in the $Z W W$ and $\gamma W W$ vertices, as given in Eq.(2.15)):

$$
\begin{aligned}
& (o)=(o)_{\xi_{W}=1}+\mathcal{V}_{\gamma f \bar{f}}^{\mu} D_{\gamma}(q) s_{w}\left(\lambda_{W}^{2} I_{4} q^{2} k_{\mu} k_{\nu}-2 \lambda_{W} I_{3}\left[q^{2} g_{\mu \nu}-(k+q)^{2} g_{\mu \nu}+k_{\mu} k_{\nu}\right]\right) \mathcal{V}_{W \nu \bar{\nu}}^{\nu} \\
& (p)=(p)_{\xi_{W}=1}-\mathcal{V}_{Z f \bar{f}}^{\mu} D_{Z}(q) c_{w}\left(\lambda_{W}^{2} I_{4} q^{2} k_{\mu} k_{\nu}-2 \lambda_{W} I_{3}\left[q^{2} g_{\mu \nu}-(k+q)^{2} g_{\mu \nu}+k_{\mu} k_{\nu}\right]\right) \mathcal{V}_{W \nu \bar{\nu}}^{\nu} \\
& (q)=(q)_{\xi_{W}=1}-\mathcal{V}_{W f \bar{f}}^{\mu} c_{w}\left(\lambda_{W}^{2} I_{4} q^{2} k_{\mu} k_{\nu}-2 \lambda_{W} I_{3}\left[q^{2} g_{\mu \nu}-(k+q)^{2} g_{\mu \nu}+k_{\mu} k_{\nu}\right]\right) D_{Z}(q) \mathcal{V}_{Z \nu \bar{\nu}}^{\mu}
\end{aligned}
$$

Notice that the parts depending on $\xi_{W}$ are again propagator-like. To further exploit this fact, we use the identities of Eq.(2.11) to rewrite the vertices $\mathcal{V}_{W f \bar{f}}^{\mu}$ and $\mathcal{V}_{W \nu \bar{\nu}}^{\nu}$ in terms of the fundamental ones, thus obtaining

$$
\begin{aligned}
& (o)=(o)_{\xi_{W}=1}+(o)_{\gamma Z} \\
& (p)=(p)_{\xi_{W}=1}+(p)_{z Z} \\
& (q)=(q)_{\xi_{W}=1}+(q)_{\gamma Z}+(q)_{z Z}
\end{aligned}
$$

with

$$
\begin{aligned}
(o)_{\gamma Z}= & -\mathcal{V}_{\gamma f \bar{f}}^{\mu} D_{\gamma}(q)\left[s_{w} c_{w}\left(\lambda_{W}^{2} D_{\gamma}^{-1}(q) I_{4} k_{\mu} k_{\nu}-2 \lambda_{W} I_{3}\left[D_{\gamma}^{-1}(q) g_{\mu \nu}-(k+q)^{2} g_{\mu \nu}+k_{\mu} k_{\nu}\right]\right)\right. \\
& \left.D_{Z}^{-1}(q)\right] \\
& D_{Z}(q) \mathcal{V}_{Z \nu \bar{\nu}}^{\nu} \\
(p)_{Z Z}= & \mathcal{V}_{Z f \bar{f}}^{\mu} D_{Z}(q)\left[c _ { w } ^ { 2 } \left(\lambda_{W}^{2}\left\{D_{Z}^{-1}(q)+M_{Z}^{2}\right\} I_{4} k_{\mu} k_{\nu}-2 \lambda_{W} I_{3}\left[D_{Z}^{-1}(q) g_{\mu \nu}+M_{Z}^{2} g_{\mu \nu}\right.\right.\right. \\
& \left.\left.\left.-(k+q)^{2} g_{\mu \nu}+k_{\mu} k_{\nu}\right]\right) D_{Z}^{-1}(q)\right] D_{Z}(q) \mathcal{V}_{z \nu \bar{\nu}}^{\nu} \\
(q)_{\gamma Z}= & -\mathcal{V}_{\gamma f \bar{f}}^{\mu} D_{\gamma}(q)\left[s _ { w } c _ { w } D _ { \gamma } ^ { - 1 } ( q ) \left(\lambda_{W}^{2}\left\{D_{Z}^{-1}(q)+M_{Z}^{2}\right\} I_{4} k_{\mu} k_{\nu}-2 \lambda_{W} I_{3}\left[D_{Z}^{-1}(q) g_{\mu \nu}+M_{Z}^{2} g_{\mu \nu}\right.\right.\right. \\
& \left.\left.\left.-(k+q)^{2} g_{\mu \nu}+k_{\mu} k_{\nu}\right]\right)\right] D_{Z}(q) \mathcal{V}_{Z \nu \bar{\nu}}^{\nu}
\end{aligned}
$$




$$
\begin{aligned}
(q)_{Z Z}= & \mathcal{V}_{Z f \bar{f}}^{\mu} D_{Z}(q)\left[c _ { w } ^ { 2 } D _ { Z } ^ { - 1 } \left(\lambda_{W}^{2}\left\{D_{Z}^{-1}(q)+M_{Z}^{2}\right\} I_{4} k_{\mu} k_{\nu}-2 \lambda_{W} I_{3}\left[D_{Z}^{-1}(q) g_{\mu \nu}+M_{Z}^{2} g_{\mu \nu}\right.\right.\right. \\
& \left.\left.\left.-(k+q)^{2} g_{\mu \nu}+k_{\mu} k_{\nu}\right]\right)\right] D_{Z}(q) \mathcal{V}_{Z \nu \bar{\nu}}^{\nu}
\end{aligned}
$$

where we have judiciously rewritten $q^{2}=D_{\gamma}^{-1}(q)$ or $q^{2}=D_{Z}^{-1}(q)+M_{z}^{2}$. Notice that $(p)_{z Z}=(q)_{z Z}$.

In addition, the remaining Abelian-like vertex graphs together with the external fermion wave function corrections give:

$$
\begin{aligned}
(s)+(t) & =[(s)+(t)]_{\xi_{W}=1}+[(s)+(t)]_{z Z} \\
(r) & =(r)_{\xi_{W}=1}+(r)_{\gamma Z} \\
(u)+(v) & =((u)+(v))_{\xi_{W}=1}+((u)+(v))_{z Z}+[(u)+(v)]_{\gamma Z}
\end{aligned}
$$

with

$$
\begin{aligned}
{[(s)+(t)]_{Z Z} } & =-\mathcal{V}_{Z f \bar{f}}^{\mu} D_{Z}(q)\left[c_{w}^{2} \lambda_{W} D_{Z}^{-1}(q) I_{1} g_{\mu \nu}\right] D_{Z}(q) \mathcal{V}_{Z \nu \bar{\nu}}^{\nu} \\
(r)_{\gamma Z} & =\mathcal{V}_{\gamma f \bar{f}}^{\mu} D_{\gamma}(q)\left[s_{w} c_{w} \lambda_{W} D_{Z}^{-1}(q) I_{1} g_{\mu \nu}\right] D_{Z}(q) \mathcal{V}_{Z \nu \bar{\nu}}^{\nu} \\
((u)+(v))_{Z Z} & =-\mathcal{V}_{Z f \bar{f}}^{\mu} D_{Z}(q)\left[c_{w}^{2} \lambda_{W} D_{Z}^{-1}(q) I_{1} g_{\mu \nu}\right] D_{Z}(q) \mathcal{V}_{Z \nu \bar{\nu}}^{\nu} \\
((u)+(v))_{\gamma Z} & =\mathcal{V}_{\gamma f \bar{f}}^{\mu} D_{\gamma}(q)\left[s_{w} c_{w} \lambda_{W} D_{\gamma}^{-1}(q) I_{1} g_{\mu \nu}\right] D_{Z}(q) \mathcal{V}_{Z \nu \bar{\nu}}^{\nu}
\end{aligned}
$$

where again all $\xi_{W}$ dependent contributions are effectively propagator-like. Notice that $[(s)+$ $(t)]_{z Z}=((u)+(v))_{z Z}$.

Next continue with the conventional self-energy diagrams. We will focus first on (a) since a big part of its gauge dependent part cancels the entire gauge dependence coming from the vertices and the boxes, given above. Straightforward calculation, using only the elementary Ward identities triggered by the action of the longitudinal parts of the internal $W$ propagators on the three-boson vertices yields:

$$
\begin{aligned}
(a)_{Z Z}= & (a)_{Z Z}^{\left(\xi_{W}=1\right)}+L_{Z Z}\left(\xi_{W}\right) \\
& -\mathcal{V}_{Z f \bar{f}}^{\mu} D_{Z}(q)\left[c_{w}^{2}\left(\lambda_{W}^{2} q^{4} I_{4} k_{\mu} k_{\nu}-2 \lambda_{W} I_{3}\left[q^{4} g_{\mu \nu}+2 q^{2} k_{\mu} k_{\nu}-2 q^{2}(k+q)^{2} g_{\mu \nu}\right]\right)\right] D_{Z}(q) \mathcal{V}_{Z \nu \bar{\nu}}^{\nu} \\
(a)_{\gamma Z}= & (a)_{\gamma Z}^{\left(\xi_{W}=1\right)}+L_{\gamma Z}\left(\xi_{W}\right) \\
& +\mathcal{V}_{\gamma f \bar{f}}^{\mu} D_{\gamma}(q)\left[s_{w} c_{w}\left(\lambda_{W}^{2} q^{4} I_{4} k_{\mu} k_{\nu}-2 \lambda_{W} I_{3}\left[q^{4} g_{\mu \nu}+2 q^{2} k_{\mu} k_{\nu}-2 q^{2}(k+q)^{2} g_{\mu \nu}\right]\right)\right] D_{Z}(q) \mathcal{V}_{Z \nu \bar{\nu}}^{\nu}
\end{aligned}
$$

with

$$
\begin{aligned}
L_{Z Z}\left(\xi_{W}\right) & =\mathcal{V}_{Z f \bar{f}}^{\mu} D_{Z}(q)\left[2 c_{w}^{2} \lambda_{W} I_{1}\left((k+q)^{4} g_{\mu \nu}-(k+q)^{2} k_{\mu} k_{\nu}\right)\right] D_{Z}(q) \mathcal{V}_{Z \nu \bar{\nu}}^{\nu} \\
L_{\gamma Z}\left(\xi_{W}\right) & =-\mathcal{V}_{\gamma f \bar{f}}^{\mu} D_{\gamma}(q)\left[2 s_{w} c_{w} \lambda_{W} I_{1}\left((k+q)^{4} g_{\mu \nu}-(k+q)^{2} k_{\mu} k_{\nu}\right)\right] D_{Z}(q) \mathcal{V}_{Z \nu \bar{\nu}}^{\nu}
\end{aligned}
$$


Notice the appearance of $I_{1}$; this term has no $q$ dependence .

Next we will rewrite the expressions containing an explicit $q^{2}$ in terms of $D_{Z}^{-1}(q)$ and $D_{\gamma}^{-1}(q)$ :

$$
\begin{aligned}
(a)_{Z Z}= & (a)_{Z Z}^{\left(\xi_{W}=1\right)}+L_{Z Z}\left(\xi_{W}\right)+R_{Z Z}\left(\xi_{W}\right) \\
& -\mathcal{V}_{Z f \bar{f}}^{\mu} D_{Z}(q)\left[c _ { w } ^ { 2 } \left(\lambda_{W}^{2} I_{4}\left\{D_{Z}^{-2}(q)+2 M_{Z}^{2} D_{Z}^{-1}(q)\right\} k_{\mu} k_{\nu}\right.\right. \\
& \left.\left.-2 \lambda_{W} I_{3}\left[D_{Z}^{-2}(q) g_{\mu \nu}+2 D_{Z}^{-1}(q)\left(k_{\mu} k_{\nu}+M_{Z}^{2} g_{\mu \nu}-(k+q)^{2} g_{\mu \nu}\right)\right]\right)\right] D_{Z}(q) \mathcal{V}_{Z \nu \bar{\nu}}^{\nu} \\
(a)_{\gamma Z}= & (a)_{\gamma Z}^{\left(\xi_{W}=1\right)}+L_{\gamma Z}\left(\xi_{W}\right)+R_{\gamma Z}\left(\xi_{W}\right) \\
& +\mathcal{V}_{\gamma f \bar{f}}^{\mu} D_{\gamma}(q)\left[s _ { w } c _ { w } \left(\lambda_{W}^{2} I_{4}\left\{D_{Z}^{-1}(q) D_{\gamma}^{-1}(q)+M_{Z}^{2} D_{\gamma}^{-1}(q)\right\} k_{\mu} k_{\nu}-2 \lambda_{W} I_{3}\left[D_{Z}^{-1}(q) D_{\gamma}^{-1}(q) g_{\mu \nu}\right.\right.\right. \\
& \left.\left.\left.+D_{\gamma}^{-1}(q)\left\{k_{\mu} k_{\nu}+M_{Z}^{2} g_{\mu \nu}-(k+q)^{2} g_{\mu \nu}\right\}+D_{Z}^{-1}(q)\left\{k_{\mu} k_{\nu}-(k+q)^{2} g_{\mu \nu}\right\}\right]\right)\right] D_{Z}(q) \mathcal{V}_{Z \nu \bar{\nu}}^{\nu}
\end{aligned}
$$

with

$$
\begin{aligned}
R_{Z Z}\left(\xi_{W}\right) & =-\mathcal{V}_{Z f \bar{f}}^{\mu} D_{Z}(q)\left[c_{w}^{2}\left(\lambda_{W}^{2} I_{4} M_{Z}^{4} k_{\mu} k_{\nu}-2 \lambda_{W} I_{3}\left\{2 M_{Z}^{2}\left(k_{\mu} k_{\nu}-(k+q)^{2} g_{\mu \nu}\right)+M_{Z}^{4} g_{\mu \nu}\right\}\right)\right] D_{Z}(q) \mathcal{V}_{Z \nu \bar{\nu}}^{\nu} \\
R_{\gamma Z}\left(\xi_{W}\right) & =-\mathcal{V}_{\gamma f \bar{f}}^{\mu} D_{\gamma}(q)\left[2 s_{w} c_{w} \lambda_{W} I_{3} M_{Z}^{2}\left(k_{\mu} k_{\nu}-(k+q)^{2} g_{\mu \nu}\right)\right] D_{Z}(q) \mathcal{V}_{Z \nu \bar{\nu}}^{\nu}
\end{aligned}
$$

Adding the contributions from the diagrams $(n),(o),(p),(q),(a)_{z Z}$, and $(a)_{\gamma Z}$ a massive cancellation takes place, and we obtain:

$$
\begin{aligned}
(n)+(o)+(p)+(q)+(a)_{z Z}+(a)_{\gamma Z}= & (n)^{\left(\xi_{W}=1\right)}+(o)^{\left(\xi_{W}=1\right)}+(p)^{\left(\xi_{W}=1\right)}+(q)^{\left(\xi_{W}=1\right)} \\
& +(a)_{Z Z}^{\left(\xi_{W}=1\right)}+(a)_{\gamma Z}^{\left(\xi_{W}=1\right)}+R_{Z Z}\left(\xi_{W}\right)+L_{Z Z}\left(\xi_{W}\right) \\
& +R_{\gamma Z}\left(\xi_{W}\right)+L_{\gamma Z}\left(\xi_{W}\right)
\end{aligned}
$$

Thus, at this point all $\xi_{W}$-dependent pieces are contained in the sum of $R_{Z Z}\left(\xi_{W}\right)+L_{z Z}\left(\xi_{W}\right)$ and $R_{\gamma Z}\left(\xi_{W}\right)+L_{\gamma Z}\left(\xi_{W}\right)$; we will next show how these terms cancel exactly against the terms given in Eq.(3.30)) and the gauge-dependent terms of the remaining self-energy diagrams.

Indeed, straightforward algebra allows one to recast the sums $R_{Z Z}\left(\xi_{W}\right)+L_{Z Z}\left(\xi_{W}\right)$ and $R_{\gamma Z}\left(\xi_{W}\right)+$ $L_{\gamma Z}\left(\xi_{W}\right)$ in the following form:

$$
\begin{aligned}
R_{Z Z}\left(\xi_{W}\right)+L_{Z Z}\left(\xi_{W}\right)= & -\mathcal{V}_{Z f \bar{f}}^{\mu} D_{Z}(q)\left[c _ { w } ^ { 2 } \left(\lambda_{W}^{2} I_{4} M_{Z}^{4} k_{\mu} k_{\nu}-2 \lambda_{W}\left\{\left(k^{2} g_{\mu \nu}-k_{\mu} k_{\nu}\right) I_{1}\right.\right.\right. \\
& +\left(2 M_{Z}^{2}-M_{W}^{2}\right) I_{3} k_{\mu} k_{\nu}+\left(M_{Z}^{2}-M_{W}^{2}\right)^{2} I_{3} g_{\mu \nu}+D_{Z}^{-1}(q) I_{1} g_{\mu \nu} \\
& \left.\left.\left.+\left(M_{W}^{2}-M_{Z}^{2}\right) I_{1} g_{\mu \nu}\right\}\right)\right] D_{Z}(q) \mathcal{V}_{Z \nu \bar{\nu}}^{\nu}
\end{aligned}
$$

and

$$
R_{\gamma Z}\left(\xi_{W}\right)+L_{\gamma Z}\left(\xi_{W}\right)=-\mathcal{V}_{\gamma_{f \bar{f}}}^{\mu} D_{\gamma}(q)\left[2 s _ { w } c _ { w } \lambda _ { W } \left\{\left(k^{2} g_{\mu \nu}-k_{\mu} k_{\nu}\right) I_{1}+\left(M_{Z}^{2}-M_{W}^{2}\right) I_{3} k_{\mu} k_{\nu}\right.\right.
$$




$$
\begin{aligned}
& +M_{W}^{2}\left(M_{W}^{2}-M_{Z}^{2}\right) I_{3} g_{\mu \nu}+\frac{1}{2} D_{Z}^{-1}(q) I_{1} g_{\mu \nu}+\frac{1}{2} D_{\gamma}^{-1}(q) I_{1} g_{\mu \nu} \\
& \left.\left.+\left(M_{W}^{2}-\frac{1}{2} M_{Z}^{2}\right) I_{1} g_{\mu \nu}\right\}\right] D_{z}(q) \mathcal{V}_{z \nu \bar{\nu}}^{\nu}
\end{aligned}
$$

One notices immediately that the terms proportional to $D_{Z}^{-1}(q)$ and $D_{\gamma}^{-1}(q)$ in Eq.(3.36)) and Eq.(3.37)) cancel exactly against the terms in Eq.(3.30)). From the rest of the self-energy diagrams, with the aid of the identity in Eq.(3.23)), we obtain

$$
\begin{aligned}
(b)_{Z Z}+(c)_{Z Z}= & (b)_{Z Z}^{\left(\xi_{W}=1\right)}+(c)_{Z Z}^{\left(\xi_{W}=1\right)} \\
& +\mathcal{V}_{z f \bar{f}}^{\mu} D_{Z}(q)\left[2 s_{w}^{4} M_{Z}^{2}\left(\lambda_{W}^{2} M_{W}^{2} I_{4} k_{\mu} k_{\nu}-\lambda_{W} I_{3}\left\{M_{W}^{2} g_{\mu \nu}+k_{\mu} k_{\nu}\right\}\right)\right] D_{Z}(q) \mathcal{V}_{Z \nu \bar{\nu}}^{\nu} \\
(d)_{Z Z}+(e)_{Z Z}= & (d)_{Z Z}^{\left(\xi_{W}=1\right)}+(e)_{Z Z}^{\left(\xi_{W}=1\right)} \\
& +\mathcal{V}_{Z f \bar{f}}^{\mu} D_{Z}(q)\left[2 c_{w}^{4} M_{Z}^{2}\left(\lambda_{W}^{2} M_{W}^{2} I_{4}-2 \lambda_{W} I_{3}\right) k_{\mu} k_{\nu}\right] D_{Z}(q) \mathcal{V}_{Z \nu \bar{\nu}}^{\nu} \\
(f)_{Z Z}= & (f)_{Z Z}^{\left(\xi_{W}=1\right)}-\mathcal{V}_{Z f \bar{f}}^{\mu} D_{Z}(q)\left[\left(c_{w}^{2}-s_{w}^{2}\right)^{2} M_{Z}^{2}\left(\lambda_{W}^{2} M_{W}^{2} I_{4}-2 \lambda_{W} I_{3}\right) k_{\mu} k_{\nu}\right] D_{Z}(q) \mathcal{V}_{Z \nu \bar{\nu}}^{\nu} \\
(g)_{Z Z}= & (g)_{Z Z}^{\left(\xi_{W}=1\right)}-\mathcal{V}_{Z f \bar{f}}^{\mu} D_{Z}(q)\left[2 \lambda_{W} c_{w}^{2}\left(k^{2} g_{\mu \nu}-k_{\mu} k_{\nu}\right) I_{1}\right] D_{Z}(q) \mathcal{V}_{Z \nu \bar{\nu}}^{\nu} \\
(h)_{Z Z}= & (h)_{Z Z}^{\left(\xi_{W}=1\right)}-\mathcal{V}_{Z f \bar{f}}^{\mu} D_{Z}(q)\left[\frac{1}{2} \lambda_{W}\left(c_{w}^{2}-s_{w}^{2}\right)^{2} M_{Z}^{2} I_{1} g_{\mu \nu}\right] D_{Z}(q) \mathcal{V}_{Z \nu \bar{\nu}}^{\nu} \\
(i)_{Z Z}= & (i)_{Z Z}^{\left(\xi_{W}=1\right)}-\mathcal{V}_{Z f \bar{f}}^{\mu} D_{Z}(q)\left[\left(\frac{M_{Z}^{2}}{M_{H}^{2}}\right) \lambda_{W} k^{2} I_{1} g_{\mu \nu}\right] D_{Z}(q) \mathcal{V}_{Z \nu \bar{\nu}}^{\nu} \\
(j)_{Z Z}= & (j)_{Z Z}^{\left(\xi_{W}=1\right)}+\mathcal{V}_{Z f \bar{f}}^{\mu} D_{Z}(q)\left[\left(\frac{M_{Z}^{2}}{M_{H}^{2}}\right) \lambda_{W} k^{2} I_{1} g_{\mu \nu}\right] D_{Z}(q) \mathcal{V}_{Z \nu \bar{\nu}}^{\nu} \\
(k)_{Z Z}= & (k)_{Z Z}^{\left(\xi_{W}=1\right)}+\mathcal{V}_{Z f \bar{f}}^{\mu} D_{Z}(q)\left[\frac{1}{2} \lambda_{W} M_{Z}^{2} I_{1} g_{\mu \nu}\right] D_{Z}(q) \mathcal{V}_{Z \nu \bar{\nu}}^{\nu}
\end{aligned}
$$

and

$$
\begin{aligned}
(b)_{\gamma Z}+(c)_{\gamma Z}= & (b)_{\gamma Z}^{\left(\xi_{W}=1\right)}+(c)_{\gamma Z}^{\left(\xi_{W}=1\right)} \\
& +\mathcal{V}_{\gamma f \bar{f}}^{\mu} D_{Z}(q)\left[2 c_{w} s_{w}^{3} M_{Z}^{2}\left(\lambda_{W}^{2} M_{W}^{2} I_{4} k_{\mu} k_{\nu}-\lambda_{W} I_{3}\left\{M_{W}^{2} g_{\mu \nu}+k_{\mu} k_{\nu}\right\}\right)\right] D_{Z}(q) \mathcal{V}_{\gamma \nu \bar{\nu}}^{\nu} \\
(d)_{\gamma Z}+(e)_{\gamma Z}= & (d)_{\gamma Z}^{\left(\xi_{W}=1\right)}+(e)_{\gamma Z}^{\left(\xi_{W}=1\right)} \\
& +\mathcal{V}_{\gamma f \bar{f}}^{\mu} D_{Z}(q)\left[2 s_{w} c_{w}^{3} M_{Z}^{2}\left(\lambda_{W}^{2} M_{W}^{2} I_{4}-2 \lambda_{W} I_{3}\right) k_{\mu} k_{\nu}\right] D_{Z}(q) \mathcal{V}_{Z \nu \bar{\nu}}^{\nu} \\
(f)_{\gamma Z}= & (f)_{\gamma Z}^{\left(\xi_{W}=1\right)}+\mathcal{V}_{\gamma f \bar{f}}^{\mu} D_{Z}(q)\left[2 c_{w} s_{w}\left(c_{w}^{2}-s_{w}^{2}\right) M_{Z}^{2}\left(\lambda_{W}^{2} M_{W}^{2} I_{4}-2 \lambda_{W} I_{3}\right) k_{\mu} k_{\nu}\right] D_{Z}(q) \mathcal{V}_{z \nu \bar{\nu}}^{\nu} \\
(g)_{\gamma Z}= & (g)_{\gamma Z}^{\left(\xi_{W}=1\right)}+\mathcal{V}_{\gamma f \bar{f}}^{\mu} D_{\gamma}(q)\left[2 \lambda_{W} s_{w} c_{w}\left(k^{2} g_{\mu \nu}-k_{\mu} k_{\nu}\right) I_{1}\right] D_{Z}(q) \mathcal{V}_{Z \nu \bar{\nu}}^{\nu} \\
(h)_{\gamma Z}= & (h)_{\gamma Z}^{\left(\xi_{W}=1\right)}+\mathcal{V}_{\gamma f \bar{f}}^{\mu} D_{\gamma}(q)\left[2 c_{w} s_{w} \lambda_{W}\left(M_{W}^{2}-\frac{1}{2} M_{Z}^{2}\right) I_{1} g_{\mu \nu}\right] D_{Z}(q) \mathcal{V}_{z \nu \bar{\nu}}^{\nu}
\end{aligned}
$$


Using that $2 s_{w}^{4}+2 c_{w}^{4}-\left(c_{w}^{2}-s_{w}^{2}\right)^{2}=1$ and that $\left(c_{w}^{2}-s_{w}^{2}\right)^{2}=1-4 s_{w}^{2} c_{w}^{2}$ one can easily verify that all remaining $\xi_{W}$-dependent terms cancel. Notice in particular that the terms proportional to $\lambda_{W}^{2}$ in $(b)_{\gamma z}+(c)_{\gamma z},(d)_{\gamma z}+(e)_{\gamma z}$, and $(f)_{\gamma Z}$, cancel among 0: each other.

\section{The polarized case in the $R_{\xi}$ gauges}

We next turn to the case where the target fermions are right-handedly polarized. As already mentioned, in that case, due to the helicity mismatch, there is no coupling of the $W$ to the fermions, and therefore there is no $W W$ box. The purpose of this section is to demonstrate how the gauge cancellation go through in the absence of the box. As we will see one arrives at precisely the same expressions for the individual propagators and vertices which couple electromagnetically to the target fermions as in the previous section. Thus, even though the aforementioned vertices and propagators will be further rearranged following the PT algorithm, it is already clear at this stage that the remaining box graphs simply cannot enter in the (process-independent) definition of the NCR.

The mechanism which enforces the gauge cancellations in the absence of the box has already been outlined at the end of section 3. The modification of the bare vertices $\Gamma_{\gamma_{f_{R}} \bar{f}_{R}}^{\mu}$ and $\Gamma_{Z f_{R} \bar{f}_{R}}^{\mu}$ given in Eq.(2.19) allows the communication between Feynman diagrams whose only difference is whether the incoming gauge boson is a photon or a $Z$ (for example the diagrams $(a)_{\gamma Z}$ and $(a)_{Z Z}$ of Fig.1, respectively). In particular, due to Eq.(2.19)) the sum of any two such graphs would cancel if it was not for the fact that the tree-level propagators connecting them to the external right-handed fermions are different $\left(D_{\gamma}(q)\right.$ for $(a)_{\gamma z}, D_{z}(q)$ for $\left.(a)_{z z}\right)$. But the gauge dependent pieces coming from these graphs are precisely proportional to inverse bare photon and $Z$ propagators, $\left(D_{\gamma}^{-1}(q)\right.$ for $(a)_{\gamma Z}, D_{Z}^{-1}(q)$ for $\left.(a)_{z Z}\right)$, a fact which allows their cancellation to be actually implemented.

We now turn to the details of the calculation. Having right-handedly polarized fermions is equivalent to saying that there are no contributions coming from the graphs $(n),(q)$, and $(u)+(v)$, since these graphs vanish identically. Next we show in detail how the cancellations of all GFP-dependent contributions take place. The general strategy is to let the propagators on the left of each parentheses hit there inverses inside the parentheses, and then employ the identity of Eq.(2.20). For example, the contributions from $(o)_{\gamma z}$ and $(p)_{z z}$ can be rewritten in the form

$$
\begin{aligned}
(o)_{\gamma Z}= & -\mathcal{V}_{\gamma f \bar{f}}^{\mu} D_{\gamma}(q)\left[s_{w} c_{w}\left(D_{\gamma}^{-1}(q)\left(\lambda_{W}^{2} I_{4} k_{\mu} k_{\nu}-2 \lambda_{W} I_{3} g_{\mu \nu}\right)-2 \lambda_{W} I_{3}\left[k_{\mu} k_{\nu}-(k+q)^{2} g_{\mu \nu}\right]\right) D_{Z}^{-1}(q)\right] \\
& D_{Z}(q) \mathcal{V}_{Z \nu \bar{\nu}}^{\nu} \\
(p)_{Z Z}= & \mathcal{V}_{Z f \bar{f}}^{\mu} D_{Z}(q)\left[c _ { w } ^ { 2 } \left(D_{Z}^{-1}(q)\left(\lambda_{W}^{2} I_{4} k_{\mu} k_{\nu}-2 \lambda_{W} I_{3} g_{\mu \nu}\right)+\lambda_{W}^{2} M_{Z}^{2} I_{4} k_{\mu} k_{\nu}-2 \lambda_{W} I_{3}\left[M_{Z}^{2} g_{\mu \nu}\right.\right.\right. \\
& \left.\left.\left.-(k+q)^{2} g_{\mu \nu}+k_{\mu} k_{\nu}\right]\right) D_{Z}^{-1}(q)\right] D_{Z}(q) \mathcal{V}_{Z \nu \bar{\nu}}^{\nu}
\end{aligned}
$$

and so in the case where the initial fermions are right-handed we have

$$
(o)_{\gamma Z}^{R R}+(p)_{Z Z}^{R R}=\mathcal{V}_{\gamma_{f_{R} \bar{f}_{R}}}^{\mu} D_{\gamma}(q)\left[s_{w} c_{w}\left(2 \lambda_{W} I_{3}\left[k_{\mu} k_{\nu}-(k+q)^{2} g_{\mu \nu}\right]\right) D_{Z}^{-1}(q)\right] D_{Z}(q) \mathcal{V}_{Z \nu \bar{\nu}}^{\nu}
$$




$$
\begin{aligned}
& +\mathcal{V}_{\gamma_{f_{R} \bar{f}_{R}}}^{\mu} D_{Z}(q)\left[s_{w} c_{w}\left(\lambda_{W}^{2} M_{Z}^{2} I_{4} k_{\mu} k_{\nu}-2 \lambda_{W} I_{3}\left[M_{Z}^{2} g_{\mu \nu}-(k+q)^{2} g_{\mu \nu}+k_{\mu} k_{\nu}\right]\right) D_{Z}^{-1}(q)\right] \\
& D_{Z}(q) \mathcal{V}_{Z \nu \bar{\nu}}^{\nu}
\end{aligned}
$$

Similarly we obtain from the Abelian-like graphs of Fig. 7

$$
\begin{aligned}
{[(s)+(t)]_{Z Z}^{R R}+(r)_{\gamma Z}^{R R}=} & -\mathcal{V}_{\gamma_{f_{R} \bar{f}_{R}}^{\mu}}^{\mu} D_{Z}(q)\left[s_{w} c_{w} \lambda_{W} D_{Z}^{-1}(q) I_{1} g_{\mu \nu}\right] D_{Z}(q) \mathcal{V}_{Z \nu \bar{\nu}}^{\nu} \\
& +\mathcal{V}_{\gamma_{f_{R} \bar{f}_{R}}^{\mu}}^{\mu} D_{\gamma}(q)\left[s_{w} c_{w} \lambda_{W} D_{Z}^{-1}(q) I_{1} g_{\mu \nu}\right] D_{Z}(q) \mathcal{V}_{Z \nu \bar{\nu}}^{\nu}
\end{aligned}
$$

Here there is no cancellation except for the simplification of writing the vertices in front using the relation of Eq.(2.20) . Turning to the self-energy graphs we have

$$
\begin{aligned}
(a)_{Z Z}^{R R}= & (a)_{Z Z}^{R R\left(\xi_{W}=1\right)}+L_{Z Z}^{R R}\left(\xi_{W}\right)+R_{Z Z}^{R R}\left(\xi_{W}\right) \\
& -\mathcal{V}_{Z f \bar{f}}^{\mu} D_{Z}(q)\left[c _ { w } ^ { 2 } \left(D_{Z}^{-1}(q) S_{\mu \nu}+\lambda_{W}^{2} M_{Z}^{2} I_{4} D_{Z}^{-1}(q) k_{\mu} k_{\nu}\right.\right. \\
& \left.\left.-2 \lambda_{W} I_{3} D_{Z}^{-1}(q)\left(k_{\mu} k_{\nu}+M_{Z}^{2} g_{\mu \nu}-(k+q)^{2} g_{\mu \nu}\right)\right)\right] D_{Z}(q) \mathcal{V}_{z \nu \bar{\nu}}^{\nu} \\
(a)_{\gamma Z}^{R R}= & (a)_{\gamma Z}^{R R\left(\xi_{W}=1\right)}+L_{\gamma Z}^{R R}\left(\xi_{W}\right)+R_{\gamma Z}^{R R}\left(\xi_{W}\right) \\
& \left.+\mathcal{V}_{\gamma f \bar{f}}^{\mu} D_{\gamma}(q)\left[s_{w} c_{w}\left(D_{\gamma}^{-1}(q) S_{\mu \nu}-2 \lambda_{W} I_{3} D_{Z}^{-1}(q)\left\{k_{\mu} k_{\nu}-(k+q)^{2} g_{\mu \nu}\right\}\right]\right)\right] D_{Z}(q) \mathcal{V}_{z \nu \bar{\nu}}^{\nu}
\end{aligned}
$$

with

$$
S_{\mu \nu}=\lambda_{W}^{2} I_{4}\left(D_{Z}^{-1}(q)+M_{Z}^{2}\right) k_{\mu} k_{\nu}-2 \lambda_{W} I_{3}\left(D_{Z}^{-1}(q) g_{\mu \nu}+k_{\mu} k_{\nu}+M_{Z}^{2} g_{\mu \nu}-(k+q)^{2} g_{\mu \nu}\right)
$$

Adding by parts we see that the terms proportional to $S_{\mu \nu}$ cancel, and we are left with

$$
\begin{aligned}
(a)_{Z Z}^{R R}+(a)_{\gamma Z}^{R R}= & (a)_{Z Z}^{R R\left(\xi_{W}=1\right)}+(a)_{\gamma Z}^{R R\left(\xi_{W}=1\right)}+L_{z Z}^{R R}\left(\xi_{W}\right)+R_{Z Z}^{R R}\left(\xi_{W}\right)+L_{\gamma Z}^{R R}\left(\xi_{W}\right)+R_{\gamma Z}^{R R}\left(\xi_{W}\right) \\
& -\mathcal{V}_{\gamma_{f} \bar{f}_{R}}^{\mu} D_{Z}(q)\left[s _ { w } c _ { w } \left(\lambda_{W}^{2} M_{Z}^{2} I_{4} D_{Z}^{-1}(q) k_{\mu} k_{\nu}\right.\right. \\
& \left.\left.-2 \lambda_{W} I_{3} D_{Z}^{-1}(q)\left(k_{\mu} k_{\nu}+M_{Z}^{2} g_{\mu \nu}-(k+q)^{2} g_{\mu \nu}\right)\right)\right] D_{Z}(q) \mathcal{V}_{z \nu \bar{\nu}}^{\nu} \\
& \left.-\mathcal{V}_{\gamma_{f_{R}} \bar{f}_{R}}^{\mu} D_{\gamma}(q)\left[s_{w} c_{w}\left(2 \lambda_{W} I_{3}\left\{k_{\mu} k_{\nu}-(k+q)^{2} g_{\mu \nu}\right\} D_{Z}^{-1}(q)\right]\right)\right] D_{Z}(q) \mathcal{V}_{z \nu \bar{\nu}}^{\nu} \\
= & (a)_{Z Z}^{R R\left(\xi_{W}=1\right)}(a)_{\gamma Z}^{R R\left(\xi_{W}=1\right)}+L_{Z Z}^{R R}\left(\xi_{W}\right)+R_{z Z}^{R R}\left(\xi_{W}\right)+L_{\gamma Z}^{R R}\left(\xi_{W}\right)+R_{\gamma Z}^{R R}\left(\xi_{W}\right) \\
& -\left((o)_{\gamma Z}^{R R}+(p)_{Z Z}^{R R}\right)
\end{aligned}
$$

At this point it is clear that one has effectively arrived at the same stage one was in the previous section right after Eq.(3.37), except for the fact that now, instead of the $\xi_{W}$-dependent contributions from $[(s)+(t)]_{z Z},(r)_{\gamma Z},((u)+(v))_{z Z},((u)+(v))_{\gamma z}$ in Eq.(3.30), we only have the 
$\xi_{W}$-dependent contributions from $[(s)+(t)]_{Z Z}^{R R}$ and $(r)_{\gamma Z}^{R R}$. Notice however that, again by virtue of Eq.(2.20), now there is an extra cancellation possible between the part in $L_{Z Z}^{R R}\left(\xi_{W}\right)+R_{Z Z}^{R R}\left(\xi_{W}\right)$ proportional to $D_{Z}^{-1}(q) I_{1}$ and the part in $L_{Z Z}^{R R}\left(\xi_{W}\right)+R_{Z Z}^{R R}\left(\xi_{W}\right)+L_{\gamma Z}^{R R}\left(\xi_{W}\right)+R_{\gamma Z}^{R R}\left(\xi_{W}\right)$ proportional to $D_{\gamma}^{-1}(q) I_{1}$, a fact which exactly compensates for the absence of ( $\xi_{W}$-dependent) contributions from $((u)+(v))_{z z},((u)+(v))_{\gamma z}$. Having realized that, then the rest of the cancellations proceed exactly as in the unpolarized case.

\section{The gauge cancellations in the Background Field Method}

The BFM [28-31] is a special gauge-fixing procedure which preserves the symmetry of the action under ordinary gauge transformations with respect to the background (classical) gauge field, while the (quantum) gauge fields appearing in the loops transform homogeneously under the gauge group, i.e. as ordinary matter fields which happened to be assigned to the adjoint representation. As happens within every gauge-fixing scheme the off-shell Green's functions derived in this formalism depend explicitly on the GFP, but due the residual background symmetry they have the advantage of being gauge-invariant, i.e. they satisfy naive QED-like Ward identities to all orders in perturbation theory, and for every value of the GFP. As has been shown in detail, when the GFP-dependent BFM Green's function are computed in the Feynman gauge they coincide with the GFP-independent PT Green's function, both at one loop [27] and at two loops [25].

In this section we will demonstrate that the GFP cancellations in the context of the BFM proceed exactly as in the case of the $R_{\xi}$ gauges, i.e. through algebraic manipulations only, provided that one exploits again the fundamental cancellation mechanism operating in the $S$-matrix element; the latter holds regardless of the gauge-fixing scheme chosen when quantizing the theory. It turns out that once the GFP cancellations have been implemented one recovers exactly the same set of Feynman diagrams one had ended up with in the context of the $R_{\xi}$ gauges once the corresponding GFP cancellation procedure had been completed, i.e. at the end of section 3. Evidently the GFP cancellation carried out using the PT rules projects us from one gauge-fixing scheme $(\mathrm{BFM})$ to another $\left(R_{\xi}\right)$. This makes abundantly clear the fact that the BFM Feynman gauge (which is different from the $R_{\xi}$ Feynman gauge) has not been singled out in any a-priori manner, since, in fact, one is effectively led to the Feynman rules of a different gauge fixing scheme. Of course, one will reach eventually the BFM in the Feynman gauge (regardless of the starting point) once the PT procedure has been completed, i.e. once the final rearrangement, described in the following section, has been carried out.

In the BFM the elementary three-boson vertex between a background field $\widetilde{A}_{\mu}(q)$ and two quantum fields $A_{\rho}\left(p_{2}\right)$ and $A_{\sigma}\left(p_{3}\right)$ for a general value of the quantum gauge-fixing parameter $\xi_{Q}^{W}$ reads $[40]$

$$
\widetilde{\Gamma}_{\mu \rho \sigma}\left(q, p_{2}, p_{3}\right)=\left(q-p_{2}-\frac{1}{\xi_{Q}^{W}} p_{3}\right)_{\sigma} g_{\mu \rho}+\left(p_{2}-p_{3}\right)_{\mu} g_{\rho \sigma}+\left(p_{3}-q+\frac{1}{\xi_{Q}^{W}} p_{2}\right)_{\rho} g_{\mu \sigma}
$$

which, inside the loop can be written as

$$
\widetilde{\Gamma}_{\mu \rho \sigma}(q, k,-k-q)=\Gamma_{\mu \rho \sigma}^{F}-\left(\frac{1-\xi_{Q}^{W}}{\xi_{Q}^{W}}\right) \Gamma_{\mu \rho \sigma}^{P}
$$


or

$$
\widetilde{\Gamma}_{\mu \rho \sigma}(q, k,-k-q)=\Gamma_{\mu \rho \sigma}(q, k,-k-q)-\frac{1}{\xi_{Q}^{W}} \Gamma_{\mu \rho \sigma}^{P}
$$

In what follows we will employ the latter decomposition. This will allow to project a large part of the calculation into effectively the $R_{\xi}$ diagrammatic formulation and expedite the demonstration of the cancellations by using directly the results of the previous section, by simply setting $\xi \rightarrow \xi_{Q}^{W}$. Similarly, the elementary four-gauge-boson vertex involving two background fields, $\widetilde{A}_{\mu}$ and $\widetilde{A}_{\nu}$, and two quantum fields, $A_{\rho}$ and $A_{\sigma}$, reads

$$
\begin{aligned}
\widetilde{\Gamma}_{\mu \nu \rho \sigma} & =2 g_{\mu \nu} g_{\rho \sigma}+\left(\frac{1-\xi_{Q}^{W}}{\xi_{Q}^{W}}\right) g_{\mu \sigma} g_{\nu \rho}+\left(\frac{1-\xi_{Q}^{W}}{\xi_{Q}^{W}}\right) g_{\mu \rho} g_{\nu \sigma} \\
& =\Gamma_{\mu \nu \rho \sigma}+\frac{1}{\xi_{Q}^{W}}\left(g_{\mu \sigma} g_{\nu \rho}+g_{\mu \rho} g_{\nu \sigma}\right)
\end{aligned}
$$

Finally, the tree-level gauge boson propagators $\widetilde{\Delta}_{\tilde{V}}^{\mu \nu}(k)$ are identical to those given in Eq.(2.1), with $\xi \rightarrow \xi_{Q}^{W}$. The strategy of the proof consists in splitting all BFM Feynman graphs as a part which is identical to the corresponding Feynman graph in the $R_{\xi}$ gauge, plus a leftover $\xi_{Q}^{W}$-dependent contribution. Then, all one has to show is that all such additional contribution cancel against each other.

To prove that this is indeed the case, let us first list the graphs which are identical to those in the $R_{\xi}$ after the trivial change $\xi \rightarrow \xi_{Q}^{W}$ :

$$
\begin{aligned}
(\widetilde{n}) & =(n) \\
(\widetilde{r}) & =(r) \\
(\widetilde{s})+(\widetilde{t}) & =(s)+(t) \\
(\widetilde{u})+(\widetilde{v}) & =(u)+(v) \\
(\widetilde{f}) & =(f) \\
(\widetilde{h}) & =(h) \\
(\widetilde{i}) & =(i) \\
(\widetilde{j}) & =(j) \\
(\widetilde{k}) & =(k)
\end{aligned}
$$

The rest of the diagrams differ from those in the $R_{\xi}$ gauge. In particular, for the vertex graphs we have

$$
\begin{aligned}
(\widetilde{o}) & =(o)+(\widetilde{o})_{\gamma Z} \\
(\widetilde{p}) & =(p)+(\widetilde{p})_{z Z} \\
(\widetilde{q}) & =(q)+(\widetilde{q})_{\gamma Z}+(\widetilde{q})_{z Z}
\end{aligned}
$$

with

$$
(\widetilde{o})_{\gamma Z}=-\mathcal{V}_{\gamma f \bar{f}}^{\mu} D_{\gamma}(q)\left[2 s_{w} c_{w} I_{5} \Delta_{\mu \nu}^{W}(k+q) D_{Z}^{-1}(q)\right] D_{Z}(q) \mathcal{V}_{Z \nu \bar{\nu}}^{\nu}
$$




$$
\begin{aligned}
(\widetilde{p})_{Z Z} & =\mathcal{V}_{Z f \bar{f}}^{\mu} D_{Z}(q)\left[2 c_{w}^{2} I_{5} \Delta_{\mu \nu}^{W}(k+q) D_{Z}^{-1}(q)\right] D_{Z}(q) \mathcal{V}_{Z \nu \bar{\nu}}^{\nu} \\
(\widetilde{q})_{\gamma Z} & =-\mathcal{V}_{\gamma f \bar{f}}^{\mu} D_{\gamma}(q)\left[2 s_{w} c_{w} D_{\gamma}^{-1}(q) I_{5} \Delta_{\mu \nu}^{W}(k+q)\right] D_{Z}(q) \mathcal{V}_{Z \nu \bar{\nu}}^{\nu} \\
(\widetilde{q})_{Z Z} & =\mathcal{V}_{Z f \bar{f}}^{\mu} D_{Z}(q)\left[2 c_{w}^{2} D_{Z}^{-1}(q) I_{5} \Delta_{\mu \nu}^{W}(k+q)\right] D_{Z}(q) \mathcal{V}_{Z \nu \bar{\nu}}^{\nu}
\end{aligned}
$$

Similarly, the self-energy graphs give

$$
\begin{aligned}
(\widetilde{a})_{z Z} & =(a)_{z Z}+G_{Z Z}\left(\xi_{Q}^{W}\right) \\
(\widetilde{a})_{\gamma Z} & =(a)_{\gamma Z}+G_{\gamma Z}\left(\xi_{Q}^{W}\right)
\end{aligned}
$$

with

$$
\begin{aligned}
G_{Z Z}\left(\xi_{Q}^{W}\right)= & \mathcal{V}_{Z f \bar{f}}^{\mu} D_{Z}(q)\left[2 c _ { w } ^ { 2 } \left(2 D_{Z}^{-1}(q) I_{5} \Delta_{\mu \nu}^{W}(k+q)+3 I_{2} k_{\mu} k_{\nu}+\left(\frac{1}{\xi_{Q}^{W}}\right) \Delta_{\mu \nu}^{W}(k)\right.\right. \\
& \left.\left.-2 I_{5} g_{\mu \nu}+\left(2 M_{Z}^{2}-M_{W}^{2}\right) I_{5} \Delta_{\mu \nu}^{W}(k+q)\right)\right] D_{Z}(q) \mathcal{V}_{Z \nu \bar{\nu}}^{\nu} \\
G_{\gamma Z}\left(\xi_{Q}^{W}\right)= & -\mathcal{V}_{\gamma f \bar{f}}^{\mu} D_{\gamma}(q)\left[2 s _ { w } c _ { w } \left(\left\{D_{\gamma}^{-1}(q)+D_{Z}^{-1}(q)\right\} I_{5} \Delta_{\mu \nu}^{W}(k+q)+3 I_{2} k_{\mu} k_{\nu}+\left(\frac{1}{\xi_{Q}^{W}}\right) \Delta_{\mu \nu}^{W}(k)\right.\right. \\
& \left.\left.-2 I_{5} g_{\mu \nu}+\left(M_{Z}^{2}-M_{W}^{2}\right) I_{5} \Delta_{\mu \nu}^{W}(k+q)\right)\right] D_{Z}(q) \mathcal{V}_{Z \nu \bar{\nu}}^{\nu}
\end{aligned}
$$

and the remaining self-energy graphs yield

$$
\begin{aligned}
(\widetilde{b})_{z Z}+(\widetilde{c})_{z Z} & =(b)_{z Z}+(c)_{Z Z}+\mathcal{V}_{Z f \bar{f}}^{\mu} D_{Z}(q)\left[2 c_{w}^{2}\left(2 M_{Z}^{2}-M_{W}^{2}\right) I_{5} \Delta_{\mu \nu}^{W}(k+q)\right] D_{Z}(q) \mathcal{V}_{Z \nu \bar{\nu}}^{\nu} \\
(\widetilde{d})_{z Z}+(\tilde{e})_{z Z} & =(d)_{z Z}+(e)_{z Z}+\mathcal{V}_{Z f \bar{f}}^{\mu} D_{Z}(q)\left[6 c_{w}^{2} I_{2} k_{\mu} k_{\nu}\right] D_{Z}(q) \mathcal{V}_{Z \nu \bar{\nu}}^{\nu} \\
(\widetilde{g})_{Z Z} & =(g)_{Z Z}+\mathcal{V}_{Z f \bar{f}}^{\mu} D_{Z}(q)\left[2 c_{w}^{2}\left(\frac{1}{\xi_{Q}^{W}}\right) \Delta_{\mu \nu}^{W}(k)\right] D_{Z}(q) \mathcal{V}_{Z \nu \bar{\nu}}^{\nu} \\
(\widetilde{\ell})_{z Z} & =\mathcal{V}_{Z f \bar{f}}^{\mu} D_{Z}(q)\left[4 c_{w}^{2} I_{5} g_{\mu \nu}\right] D_{Z}(q) \mathcal{V}_{Z \nu \bar{\nu}}^{\nu}
\end{aligned}
$$

and

$$
\begin{aligned}
(\widetilde{b})_{\gamma Z}+(\widetilde{c})_{\gamma Z} & =0=(b)_{\gamma Z}+(c)_{\gamma Z}+\mathcal{V}_{\gamma f \bar{f}}^{\mu} D_{\gamma}(q)\left[2 s_{w} c_{w}\left(M_{Z}^{2}-M_{W}^{2}\right) I_{5} \Delta_{\mu \nu}^{W}(k+q)\right] D_{Z}(q) \mathcal{V}_{Z \nu \bar{\nu}}^{\nu} \\
(\widetilde{d})_{\gamma Z}+(\widetilde{e})_{\gamma Z} & =(d)_{\gamma Z}+(e)_{\gamma Z}+\mathcal{V}_{\gamma f \bar{f}}^{\mu} D_{\gamma}(q)\left[6 s_{w} c_{w} I_{2} k_{\mu} k_{\nu}\right] D_{Z}(q) \mathcal{V}_{Z \nu \bar{\nu}}^{\nu} \\
(\widetilde{g})_{\gamma Z} & =(g)_{\gamma Z}+\mathcal{V}_{\gamma f \bar{f}}^{\mu} D_{\gamma}(q)\left[2 s_{w} c_{w}\left(\frac{1}{\xi_{Q}^{W}}\right) \Delta_{\mu \nu}^{W}(k)\right] D_{Z}(q) \mathcal{V}_{Z \nu \bar{\nu}}^{\nu}
\end{aligned}
$$


Adding Eq.(5.5)-Eq.(5.11), by parts it is straightforward to verify that all additional contributions cancel and one recovers simply the entire $S$-matrix element, expressed now as a sum of the $R_{\xi^{-}}$ gauge Feynman diagrams. Notice again that no integration over virtual momenta have been carried out .

To see how the above cancellation proceeds in the polarized case, simply notice that in that case (i) $(\widetilde{q})_{\gamma z}=(\widetilde{q})_{z z}=0$ since the diagram $(q)$ is identically zero in this case; (ii) half of the term in $G_{Z Z}\left(\xi_{Q}^{W}\right)$ proportional to $D_{z}^{-1}(q)$ and the term in $G_{\gamma z}\left(\xi_{Q}^{W}\right)$ proportional to $D_{\gamma}^{-1}(q)$ cancel against each other; (iii) finally, the remaining term in $G_{Z Z}\left(\xi_{Q}^{W}\right)$ proportional to $D_{Z}^{-1}(q)$ cancels against $(\widetilde{p})_{z Z}$ and the term in $G_{\gamma z}\left(\xi_{Q}^{W}\right)$ proportional to $D_{Z}^{-1}(q)$ cancels against $(\widetilde{o})_{\gamma z}$. The rest of the cancellation proceeds as in the unpolarized case.

The construction presented above is in a way complementary to that presented in [41]; there it was shown that the judicious use of tree-level WI allows one to to start from the Feynman diagrams written in the $R_{\xi}$ gauges and project oneself into the BFM set of Feynman diagrams for any choice of the BFM GFP. Here the reverse has been shown: one may start from the Feynman rules of the BFM (in any GFP) and, by eliminating longitudinal momenta, end up in the $R_{\xi}$ gauges. Notice that the freedom in moving diagrammatically from one gauge fixing scheme to the next does not mean that all gauges are physically equivalent; indeed, as has been explained in detail in the literature [23,24], it is only in the context of the PT, i.e. once all (and not only some) of the longitudinal momenta have been eliminated by virtue of the WI that one arrives at a physically meaningful separation of the original amplitude into self-energy, vertex, and box-like sub-amplitudes.

\section{The final rearrangement}

It is known that, when the remaining longitudinal momenta stemming from the decomposition of the vertex described in Eq.(2.3) - Eq.(2.4) are allowed to trigger the same WI as their counterparts originating from the tree-level propagators, the final one-loop answer reorganizes itself further into effective one-loop self-energies,vertices and boxes which, in addition to being GFP-independent, are gauge-invariant, i.e. they satisfy naive, QED-like WI [19-21]. To see that one simply employs the vertex decomposition given in Eq.(2.3) - Eq.(2.4) inside vertex diagrams containing a trilinear vertex, and reassigns the propagator-like contributions which emerge to the conventional self-energy graphs, thus constructing the one-loop PT self-energy $\widehat{\Pi}_{\mu \nu}$. The remaining genuine vertex graphs constitute the effective one-loop PT vertex $\widehat{\Gamma}_{\mu}$.

In what follows we will use hats to denote the individual PT Feynman diagrams emerging after this final rearrangement has been carried out. Clearly,

$$
\begin{aligned}
(\widehat{n}) & =(n)_{\left(\xi_{W}=1\right)}=(\widetilde{n})_{\left(\xi_{Q}^{W}=1\right)} \\
(\widehat{r}) & =(r)_{\left(\xi_{W}=1\right)}=(\widetilde{r})_{\left(\xi_{Q}^{W}=1\right)} \\
(\widehat{s}) & =(s)_{\left(\xi_{W}=1\right)}=(\widetilde{s})_{\left(\xi_{Q}^{W}=1\right)} \\
(\widehat{t}) & =(t)_{\left(\xi_{W}=1\right)}=(\widetilde{t})_{\left(\xi_{Q}^{W}=1\right)} \\
(\widehat{u}) & =(u)_{\left(\xi_{W}=1\right)}=(\widetilde{u})_{\left(\xi_{Q}^{W}=1\right)} \\
(\widehat{v}) & =(v)_{\left(\xi_{W}=1\right)}=(\widetilde{v})_{\left(\xi_{Q}^{W}=1\right)}
\end{aligned}
$$


The first relation in the above equation simply states that the PT box is equal to the conventional one in the Feynman gauge, both in the $R_{\xi}$ gauges and in the BFM.

From the vertex graphs containing tri-linear vertices we have:

$$
\begin{aligned}
(\widehat{o}) & =(o)_{\left(\xi_{W}=1\right)}-(o)_{\gamma Z}^{\Gamma^{P}}=(\widetilde{o})_{\left(\xi_{Q}^{W}=1\right)} \\
(\widehat{p}) & =(p)_{\left(\xi_{W}=1\right)}-(p)_{Z Z}^{\Gamma^{P}}=(\widetilde{p})_{\left(\xi_{Q}^{W}=1\right)} \\
(\widehat{q}) & =(q)_{\left(\xi_{W}=1\right)}-\left\{(q)_{Z Z}^{\Gamma^{P}}+(q)_{\gamma Z}^{\Gamma^{P}}\right\}=(\widetilde{q})_{\left(\xi_{Q}^{W}=1\right)}
\end{aligned}
$$

where

$$
\begin{aligned}
(o)_{\gamma Z}^{\Gamma^{P}} & =-2 \mathcal{V}_{\gamma f \bar{f}}^{\mu} D_{\gamma}(q)\left[s_{w} c_{w} I_{0} D_{Z}^{-1}(q)\right] D_{Z}(q) \mathcal{V}_{Z \nu \bar{\nu}}^{\nu} \\
(p)_{Z Z}^{\Gamma^{P}} & =2 \mathcal{V}_{Z f \bar{f}}^{\mu} D_{Z}(q)\left[c_{w}^{2} I_{0} D_{Z}^{-1}(q)\right] D_{Z}(q) \mathcal{V}_{Z \nu \bar{\nu}}^{\nu} \\
(q)_{\gamma Z}^{\Gamma^{P}} & =-2 \mathcal{V}_{\gamma f \bar{f}}^{\mu} D_{\gamma}(q)\left[s_{w} c_{w} D_{\gamma}^{-1}(q) I_{0}\right] D_{Z}(q) \mathcal{V}_{Z \nu \bar{\nu}}^{\nu} \\
(q)_{Z Z}^{\Gamma^{P}} & =2 \mathcal{V}_{Z f \bar{f}}^{\mu} D_{Z}(q)\left[c_{w}^{2} D_{Z}^{-1}(q) I_{0}\right] D_{Z}(q) \mathcal{V}_{Z \nu \bar{\nu}}^{\nu}
\end{aligned}
$$

are the propagator-like parts emerging after the longitudinal momenta composing $\Gamma_{\alpha \mu \nu}^{P}$ (see Eq.(2.4)) have triggered the WI of Eq.(2.6). Evidently the effective proper one-loop photonneutrino vertex $\widehat{\Gamma}_{\gamma \nu \bar{\nu}}^{\mu}$ and the PT one-loop $Z$-neutrino vertex $\widehat{\Gamma}_{z \nu \bar{\nu}}^{\mu}$ are given by

$$
\begin{aligned}
\mathcal{V}_{\gamma f \bar{f}}^{\nu} g_{\nu \mu} D_{\gamma}(q) \widehat{\Gamma}_{\gamma \nu \bar{\nu}}^{\mu} & =(\widetilde{o})_{\left(\xi_{Q}^{W}=1\right)}+(\widetilde{r})_{\left(\xi_{Q}^{W}=1\right)} \\
\mathcal{V}_{Z f \bar{f}}^{\nu} g_{\nu \mu} D_{Z}(q) \widehat{\Gamma}_{Z \nu \bar{\nu}}^{\mu} & =(\widetilde{p})_{\left(\xi_{Q}^{W}=1\right)}+(\widetilde{s})_{\left(\xi_{Q}^{W}=1\right)}+(\widetilde{t})_{\left(\xi_{Q}^{W}=1\right)}
\end{aligned}
$$

Finally, the PT self-energies $\widehat{\Pi}_{\mu \nu}^{z Z}(q)$ and $\widehat{\Pi}_{\mu \nu}^{\gamma z}(q)$ are simply the sum of all remaining self-energylike contributions, i.e.

$$
\begin{aligned}
& \mathcal{V}_{Z f \bar{f}}^{\mu} D_{Z}(q)\left[\widehat{\Pi}_{\mu \nu}^{Z Z}(q)\right] D_{Z}(q) \mathcal{V}_{Z \nu \bar{\nu}}^{\nu}=\mathcal{V}_{Z f \bar{f}}^{\mu} D_{Z}(q)\left[\Pi_{\mu \nu}^{Z Z}\left(q, \xi_{W}=1\right)\right] D_{Z}(q) \mathcal{V}_{Z \nu \bar{\nu}}^{\nu}+(p)_{Z Z}^{\Gamma_{Z}^{P}}+(q)_{Z Z}^{\Gamma^{P}} \\
& \mathcal{V}_{\gamma f \bar{f}}^{\mu} D_{\gamma}(q)\left[\widehat{\Pi}_{\mu \nu}^{Z Z}(q)\right] D_{Z}(q) \mathcal{V}_{Z \nu \bar{\nu}}^{\nu}=\mathcal{V}_{\gamma f \bar{f}}^{\mu} D_{\gamma}(q)\left[\Pi_{\mu \nu}^{\gamma Z}\left(q, \xi_{W}=1\right)\right] D_{Z}(q) \mathcal{V}_{Z \nu \bar{\nu}}^{\nu}+(o)_{\gamma Z}^{\Gamma^{P}}+(q)_{\gamma Z}^{\Gamma^{P}}
\end{aligned}
$$

It is a matter of straightforward algebra to prove that in fact

$$
\begin{aligned}
& \widehat{\Pi}_{\mu \nu}^{z Z}(q)=\widetilde{\Pi}_{\mu \nu}^{z Z}\left(q, \xi_{Q}^{W}=1\right) \\
& \widehat{\Pi}_{\mu \nu}^{\gamma Z}(q)=\widetilde{\Pi}_{\mu \nu}^{\gamma Z}\left(q, \xi_{Q}^{W}=1\right)
\end{aligned}
$$

To see that in detail, use the identity

$$
\Gamma_{\mu \rho \sigma} \Gamma_{\nu}^{\rho \sigma}=\Gamma_{\mu \rho \sigma}^{F} \Gamma_{\nu}^{F \rho \sigma}+\Gamma_{\mu \rho \sigma}^{P} \Gamma_{\nu}^{\rho \sigma}+\Gamma_{\mu \rho \sigma} \Gamma_{\nu}^{P \rho \sigma}-\Gamma_{\mu \rho \sigma}^{P} \Gamma_{\nu}^{P \rho \sigma}
$$

to cast the diagrams $(a)_{Z Z}^{\left(\xi_{W}=1\right)}$ and $(a)_{\gamma Z}^{\left(\xi_{W}=1\right)}$ in the form

$$
\begin{aligned}
& (a)_{Z Z}^{\left(\xi_{W}=1\right)}=(\widetilde{a})_{Z Z}^{\left(\xi_{Q}^{W}=1\right)}+G_{Z Z}^{\Gamma^{P}} \\
& (a)_{\gamma Z}^{\left(\xi_{W}=1\right)}=(\widetilde{a})_{\gamma Z}^{\left(\xi_{Q}^{W}=1\right)}+G_{\gamma Z}^{\Gamma^{P}}
\end{aligned}
$$


with

$$
\begin{aligned}
G_{Z Z}^{\Gamma^{P}}= & -\mathcal{V}_{z f \bar{f}}^{\mu} D_{Z}(q)\left[2 c_{w}^{2}\left(2 D_{Z}^{-1}(q) I_{0} g_{\mu \nu}+3 I_{0} k_{\mu} k_{\nu}-D_{W}(k) g_{\mu \nu}+\left(2 M_{z}^{2}-M_{W}^{2}\right) I_{0}\right)\right] D_{Z}(q) \mathcal{V}_{z \nu \bar{\nu}}^{\nu} \\
G_{\gamma Z}^{\Gamma^{P}}= & \mathcal{V}_{\gamma f \bar{f}}^{\mu} D_{\gamma}(q)\left[2 s_{w} c_{w}\left(\left\{D_{\gamma}^{-1}(q)+D_{z}^{-1}(q)\right\} I_{0} g_{\mu \nu}+3 I_{0} k_{\mu} k_{\nu}-D_{W}(k) g_{\mu \nu}+\left(M_{z}^{2}-M_{W}^{2}\right) I_{0} g_{\mu \nu}\right)\right] \\
& D_{z}(q) \mathcal{V}_{z \nu \bar{\nu}}^{\nu}
\end{aligned}
$$

Note that $G_{Z Z}^{\Gamma^{P}}=G_{Z Z}\left(\xi_{Q}^{W}=1\right)$ and $G_{\gamma Z}^{\Gamma^{P}}=G_{\gamma Z}\left(\xi_{Q}^{W}=1\right)$ Then, simple algebra yields for the remaining graphs:

$$
\begin{aligned}
(b)_{Z Z}^{\left(\xi_{W}=1\right)}+(c)_{Z Z}^{\left(\xi_{W}=1\right)} & =(\widetilde{b})_{Z Z}^{\left(\xi_{Q}^{W}=1\right)}+(\widetilde{c})_{Z Z}^{\left(\xi_{Q}^{W}=1\right)}+\mathcal{V}_{Z f \bar{f}}^{\mu} D_{Z}(q)\left[2 c_{w}^{2}\left(2 M_{Z}^{2}-M_{W}^{2}\right) I_{0} g_{\mu \nu}\right] D_{Z}(q) \mathcal{V}_{Z \nu \bar{\nu}}^{\nu} \\
(d)_{Z Z}^{\left(\xi_{W}=1\right)}+(e)_{Z Z}^{\left(\xi_{W}=1\right)} & =(\widetilde{d})_{Z Z}^{\left(\xi_{Q}^{W}=1\right)}+(\widetilde{e})_{Z Z}^{\left(\xi_{Z}^{W}=1\right)}+\mathcal{V}_{Z f \bar{f}}^{\mu} D_{Z}(q)\left[6 c_{w}^{2} I_{0} k_{\mu} k_{\nu}\right] D_{Z}(q) \mathcal{V}_{Z \nu \bar{\nu}}^{\nu} \\
(g)_{Z Z}^{\left(\xi_{W}=1\right)} & =(\widetilde{g})_{Z Z}^{\left(\xi_{Q}^{W}=1\right)}-\mathcal{V}_{Z f \bar{f}}^{\mu} D_{Z}(q)\left[2 c_{w}^{2} D_{W}(k)\right] D_{Z}(q) \mathcal{V}_{Z \nu \bar{\nu}}^{\nu}
\end{aligned}
$$

and

$$
\begin{aligned}
(b)_{\gamma Z}^{\left(\xi_{W}=1\right)}+(c)_{\gamma Z}^{\left(\xi_{W}=1\right)} & =-\mathcal{V}_{\gamma f \bar{f}}^{\mu} D_{\gamma}(q)\left[2 s_{w} c_{w}\left(M_{Z}^{2}-M_{W}^{2}\right) I_{0} g_{\mu \nu}\right] D_{Z}(q) \mathcal{V}_{Z \nu \bar{\nu}}^{\nu} \\
(d)_{\gamma Z}^{\left(\xi_{W}=1\right)}+(e)_{\gamma Z}^{\left(\xi_{W}=1\right)} & =(\widetilde{d})_{\gamma Z}^{\left(\xi_{Q}^{W}=1\right)}+(\widetilde{e})_{\gamma Z}^{\left(\xi_{Q}^{W}=1\right)}+\mathcal{V}_{\gamma f \bar{f}}^{\mu} D_{\gamma}(q)\left[6 s_{w} c_{w} I_{0} k_{\mu} k_{\nu}\right] D_{Z}(q) \mathcal{V}_{z \nu \bar{\nu}}^{\nu} \\
(g)_{\gamma Z}^{\left(\xi_{W}=1\right)} & =(\widetilde{g})_{\gamma Z}^{\left(\xi_{Q}^{W}=1\right)}+\mathcal{V}_{\gamma f \bar{f}}^{\mu} D_{\gamma}(q)\left[2 s_{w} c_{w} D_{W}(k)\right] D_{Z}(q) \mathcal{V}_{z \nu \bar{\nu}}^{\nu}
\end{aligned}
$$

Summing by parts Eq.(6.8) and Eq.(6.10) - Eq.(6.11), separating the $Z Z$ from the $\gamma Z$ components one arrives at Eq.(6.6).

The final rearrangement in the polarized case proceeds as follows: (i) $(q)_{z z}^{\Gamma^{P}}=(q)_{\gamma z}^{\Gamma^{P}}=0$; (ii) half of the term in $G_{z z}^{\Gamma^{P}}$ proportional to $D_{z}^{-1}(q)$ and the term in $G_{\gamma z}^{\Gamma^{P}}$ proportional to $D_{\gamma}^{-1}(q)$ cancel against each other; (iii) the remaining term in $G_{Z Z}^{\Gamma^{P}}$ proportional to $D_{Z}^{-1}(q)$ cancels against $(p)_{Z Z}^{\Gamma^{P}}$, and the term in $G_{\gamma z}^{\Gamma^{P}}$ proportional to $D_{Z}^{-1}(q)$ cancels against $(o)_{\gamma z}^{\Gamma^{P}}$.

\section{Results}

In this section we present closed expressions for the NCR obtained from the one-loop proper PT vertex $\widehat{\Gamma}_{\gamma \nu \bar{\nu}}^{\mu}$ given in Eq.(6.4), as well as the slope at $q^{2}=0$ of the effective self-energy $\widehat{\Pi}_{\mu \nu}^{\gamma Z}(q)$ defined in Eq.(6.6). The latter quantity has been traditionally considered as part of the NCR ; however recently it has been realized [24] that it can instead be interpreted as part of the effective mixing angle $\sin _{W, e f f}^{2}\left(q^{2}\right)$. This quantity, together with the effective charge originating from the $Z Z$ propagator, contains the universal (process-independent) corrections of every neutral 
current process. At the theoretical level the separation appears to be rather appealing, and the experimental disentanglement of these two contributions will be a matter of further study.

We begin with the transverse PT self-energy $\widehat{\Pi}_{\mu \nu}^{\gamma z}(q)$; it may be written in the form

$$
\widehat{\Pi}_{\mu \nu}^{\gamma Z}(q)=\left(g_{\mu \nu}-\frac{q_{\mu} q_{\nu}}{q^{2}}\right) \widehat{\Sigma}^{\gamma z}(q)
$$

The scalar self-energy $\widehat{\Sigma}^{\gamma z}(q)$ consists of a fermionic and a bosonic part, i.e.

$$
\widehat{\Sigma}^{\gamma z}(q)=\widehat{\Sigma}_{f}^{\gamma z}(q)+\widehat{\Sigma}_{b}^{\gamma z}(q)
$$

given by

$$
\widehat{\Sigma}_{f}^{\gamma Z}(q)=-\frac{\alpha}{6 \pi} \sum_{f} N_{f}^{c}\left(-Q_{f}\right)\left[C_{f}^{R}+C_{f}^{L}\right]\left[\frac{1}{3} q^{2}+2 m_{f}^{2} B_{0}\left(0 ; m_{f}^{2}, m_{f}^{2}\right)-\left(q^{2}+2 m_{f}^{2}\right) B_{0}\left(q^{2} ; m_{f}^{2}, m_{f}^{2}\right)\right]
$$

where

$$
\alpha=\frac{e^{2}}{4 \pi}, \quad C_{f}^{R}=-\frac{s_{W}}{c_{W}} Q_{f} \quad, \quad C_{f}^{L}=\frac{T_{z}^{F}-s_{W}^{2} Q_{f}}{s_{W} c_{W}}, \quad N_{f}^{c}=\left\{\begin{array}{c}
3 \text { quarks } \\
1 \text { leptons }
\end{array}\right.
$$

and

$$
\begin{aligned}
\widehat{\Sigma}_{b}^{\gamma z}(q)= & \frac{\alpha}{12 s_{W} c_{W} \pi}\left\{\left[\left(21 c_{W}^{2}+\frac{1}{2}\right) q^{2}+\left(12 c_{W}^{2}-2\right) M_{W}^{2}\right] B_{0}\left(q^{2} ; M_{W}^{2}, M_{W}^{2}\right)\right. \\
& \left.-\left(12 c_{W}^{2}-2\right) M_{W}^{2} B_{0}\left(0 ; M_{W}^{2}, M_{W}^{2}\right)+\frac{1}{3} q^{2}\right\}
\end{aligned}
$$

$B_{0}$ is the standard Passarino-Veltman two-point function. The counterterm corresponding to $\widehat{\Sigma}^{\gamma Z}(q)$ is given by

$$
\begin{aligned}
\frac{1}{2} \delta Z_{\gamma Z}= & -\Re e \frac{\widehat{\Sigma}^{\gamma Z}\left(M_{Z}^{2}\right)}{M_{Z}^{2}} \\
= & \frac{\alpha}{4 \pi}\left\{\frac{2}{3} \sum_{f} N_{f}^{c}\left(-Q_{f}\right)\left[C_{f}^{R}+C_{f}^{L}\right]\left[\frac{1}{3}-\left(1+2 c_{W}^{2} x_{f}\right) \beta_{f}-B_{0}\left(0 ; m_{f}^{2}, m_{f}^{2},\right)\right]\right. \\
& \left.\frac{-1}{3 s_{W} c_{W}}\left[\frac{1}{3}+\left(21 c_{W}^{2}+\frac{1}{2}\right) B_{0}\left(0 ; M_{W}^{2}, M_{W}^{2}\right)+\left(12 c_{W}^{4}+19 c_{W}^{2}+\frac{1}{2}\right) \beta_{b}\right]\right\}
\end{aligned}
$$

where

$$
\begin{aligned}
& x_{f}=\frac{m_{f}^{2}}{M_{W}^{2}} \\
& \beta_{f}=2\left\{1-\sqrt{4 c_{W}^{2} x_{f}-1} \tan ^{-1}\left(\frac{1}{\sqrt{4 c_{W}^{2} x_{f}-1}}\right)\right\} \\
& \beta_{b}=2\left\{1-\sqrt{4 c_{W}^{2}-1} \tan ^{-1}\left(\frac{1}{\sqrt{4 c_{W}^{2}-1}}\right)\right\}
\end{aligned}
$$


Thus, the renormalized derivative at the origin is given by

$$
\begin{aligned}
-\frac{\widehat{\Sigma}_{R}^{\gamma Z^{\prime}}(0)}{M_{Z}^{2}}= & \frac{3 G_{F}}{2 \pi^{2} \sqrt{2}}\left\{\frac{2}{3} \sum_{f} N_{f}^{c}\left(-Q_{f}\right)\left[C_{f}^{R}+C_{f}^{L}\right]\left[s_{W}\left(-\frac{1}{3}+\left[1+2 c_{W}^{2} x_{f}\right] \beta_{f}\right)\right]\right. \\
& \left.+\frac{1}{3 c_{W}}\left[-\left(2 c_{W}^{2}-\frac{1}{3}\right)+\left(c_{W}^{2}\left(12 c_{W}^{2}+19\right)+\frac{1}{2}\right) \beta_{b}\right]\right\} .
\end{aligned}
$$

where $G_{F}$ is the Fermi constant. Evaluating this last expression, summing over all quarks and leptons, we obtain

$$
-\frac{\widehat{\Sigma}_{R}^{\gamma Z^{\prime}}(0)}{M_{Z}^{2}}=1.6 \times 10^{-32} \mathrm{~cm}^{2} .
$$

Next we turn to the proper vertex. The Dirac form-factor $F_{1}\left(q^{2}\right)$ is usually defined as the cofactor of the $\gamma^{\mu}$ in the Lorentz decomposition of $\widehat{\Gamma}_{\gamma \nu \bar{\nu}}^{\mu}$. An elementary calculation of the two diagrams contributing to $\widehat{\Gamma}_{\gamma \nu \bar{\nu}}^{\mu}$ yields

$$
\begin{aligned}
F_{1}\left(q^{2}\right)= & -\frac{\alpha e}{8 \pi s_{W}^{2}}\left\{1+\left(\frac{1}{2}+\frac{M_{W}^{2}}{q^{2}}\right)\left[B_{0}\left(q^{2} ; m_{l}^{2}, m_{l}^{2}\right)-B_{0}\left(q^{2} ; M_{W}^{2}, M_{W}^{2}\right)\right]\right. \\
& \left.+M_{W}^{2}\left(2+\frac{M_{W}^{2}}{q^{2}}\right) C_{0}\left(0, q^{2}, 0 ; m_{l}^{2}, M_{W}^{2}, M_{W}^{2}\right)+\frac{\left(q^{2}+M_{W}^{2}\right)^{2}}{q^{2}} C_{0}\left(0, q^{2}, 0 ; M_{W}^{2}, m_{l}^{2}, m_{l}^{2}\right)\right\},
\end{aligned}
$$

where $C_{0}$ is the Passarino-Veltman three-point function, and $m_{l}$ is the mass of the charged isodoublet partner of the neutrino under consideration.

In the limit $q^{2} \rightarrow 0, F_{1}=-\frac{q^{2}}{6}<r_{\nu}^{2}>_{1}$ with

$$
\left\langle r_{\nu}^{2}\right\rangle_{1}=\frac{G_{F}}{4 \sqrt{2} \pi^{2}}\left[3-2 \log \left(\frac{m_{l}^{2}}{M_{W}^{2}}\right)\right]
$$

The numerical evaluation of the above expression for the three different neutrino species yields:

$$
\left\langle r_{\nu}^{2}\right\rangle_{1}=\left\{\begin{array}{ll}
4.1 \times 10^{-33} \mathrm{~cm}^{2} & \text { for } \nu_{e} \\
2.4 \times 10^{-33} \mathrm{~cm}^{2} & \text { for } \nu_{\mu} \\
1.5 \times 10^{-33} \mathrm{~cm}^{2} & \text { for } \nu_{\tau}
\end{array} .\right.
$$

For a spin $\frac{1}{2}$ particle, like the neutrino, the electric Sachs form-factor $G_{E}\left(q^{2}\right)$ is given in terms of the Dirac and Pauli form-factors as

$$
G_{E}\left(q^{2}\right)=F_{1}\left(q^{2}\right)+\frac{q^{2}}{4 m_{\nu}^{2}} F_{2}\left(q^{2}\right)
$$

with $F_{1}(0)=0$ and $F_{2}(0)=\mu_{\nu}$, where $\mu_{\nu}$ is the anomalous magnetic moment of the neutrino. To leading order in the mass of the Dirac neutrino, the value of $\mu_{\nu}$ is given by [16]

$$
\mu_{\nu}=\frac{3 m_{\nu}^{2} G_{F}}{4 \sqrt{2} \pi^{2}} .
$$

Its contribution (the so-called Foldy term [42]) to the charged radius is thus

$$
\begin{aligned}
\left\langle r_{\nu}^{2}\right\rangle_{2} & =-\frac{9 G_{F}}{8 \sqrt{2} \pi^{2}} \\
& =-3.7 \times 10^{-34} \mathrm{~cm}^{2}
\end{aligned}
$$




\section{Conclusions and Outlook}

This work has addressed the problem of correctly defining in the context of the Standard Model the one-loop neutrino charge radius. The discussion of this topic has a long history and it was attacked along its path with the philosophy of making "proposals". In this work we have presented a definite solution to this problem by resorting to the well-defined electroweak gaugeinvariant separation of a physical amplitude into "effective" (as opposed to "diagrammatic") selfenergy, vertex and box descompositions, implemented within the pinch technique formalism. These effective Green's function are completely independent of the gauge-fixing parameter regardless of the gauge-fixing scheme chosen. This latter property has been demonstrated explicitly by considering two very different gauge-fixing schemes, i.e. the renormalizable gauge $R_{\xi}$-scheme and the background field method. In addition, the pinch technique "effective" Green's functions satisfy simple, QED-like Ward identities which replace the involved Slavnov-Taylor identities. In the course of the derivation, we have explained in detail that the solution of the problem relies on the proper identification of the longitudinal degrees of freedom, and in particular those associated with the non-abelian character of the theory (tri-linear vertices). In addition, we have elaborated on the tree-level origin of the one-loop cancellation mechanism, especially its realization in the absence of $t$-channel tree-level graphs, i.e. in the case of $W$-pair production by right-handed target fermions.

The conceptual requirement that the effective electromagnetic vertex of a particle has to be process-independent, i. e., independent of the target used to probe the properties of the particle, is automatically implemented in our solution. In fact, the right-handedly polarized electrons provide the dynamical implementation of the earlier claim [18] that the effective boxes should not enter in the definition of the neutrino charge radius. When studying the neutrino charge form-factor, we have compared the effective neutrino vertex obtained as seen by both unpolarized electrons and right-handedly polarized electrons. As it has to be, the effective vertex is the same. However, in terms of diagrams this common result is obtained from vastly different contributions in both cases: in particular, for unpolarized electrons there is a $W W$ box contribution to the $S$-matrix, which is absent in the latter case. We have thus a neutrino charge radius which is independent of the gauge fixing parameter or the gauge-fixing scheme, couples electromagnetically to the external probe and is completely independent of it. The resulting one-loop expression for the NCR given in Eq.(7.11) depends only on the mass of the associated charged lepton.

Equipped with this theoretical identification, one can address the question of the observable character of each one of the separated effective contributions to a given process. A positive answer to this question would imply that the sub-amplitudes thus constructed not only are endowed with nice theoretical properties but are in fact physical. For example, it is known that the electroweak effective charge can be reconstructed from specific, separable contributions of the physical differential cross-section describing $W$-pair production [24]. A similar study is now in progress for the neutrino charge radius, making use of the opportunities offered by neutrino and antineutrino cross-sections and by the different neutrino flavours to take appropriate ratios.

It goes without saying that the study presented here for the neutrino charge radius can be extended to all $q^{2}$-values (either space-like or time-like) of the charge form-factor, and to all the other form-factors of the electromagnetic effective vertex. 


\section{Acknowledgments}

L.G.C.R. is indebted to the Universidad de Valencia, for his fellowhship. This research was supported by CICYT, Spain, under Grant AEN-99/0692. 


\section{References}

[1] W. Bardeen, R. Gastmans, B. Lautrup, Nucl. Phys. B46, 319 (1972).

[2] E. S. Abers, B. W. Lee, Phys. Rep. 9C, 1 (1973).

[3] K. Fujikawa, B. W. Lee, A. I. Sanda, Phys. Rev. D6, 2923 (1972).

[4] S. Y. Lee, Phys. Rev. D6, 1701 (1972).

[5] B. W. Lee, R. E. Shrock, Phys. Rev. D16, 1444 (1977).

K. Fujikawa, R. E. Shrock, Phys. Rev. Lett. 45,12 962 (1980).

R.E. Shrock, Nucl. Phys. B206, 359 (1982).

[6] J. L. Lucio, A. Rosado, A. Zepeda, Phys. Rev. D29, 1539 (1984).

N.M. Monyonko, J.H. Reid, A. Sen, Phys. Lett. 136 B, 265 (1984).

[7] J. L. Lucio, A. Rosado, A. Zepeda, Phys. Rev. D31, 1091 (1985).

[8] N.M. Monyonko, J.H. Reid, Prog. Theor. Phys. 73, 734 (1985).

[9] A. Grau and J. A. Grifols, Phys. Lett. 166B, 233 (1986).

[10] G. Auriema, Y. Srivastava, and A. Widom, Phys. Lett. 195 B, 254 (1987).

[11] J. A. Grifols and A. Grau, Mod. Phys. Lett. A Vol. 2, 205 (1987).

[12] P. Vogel and J. Engel, Phys. Rev. D 39, 3378 (1989).

[13] J. A. Grifols and E. Massó, Phys. Rev. D 40, 3819 (1989).

[14] M. J. Musolf and B. R. Holstein, Phys. Rev. D43, 2956 (1991).

[15] K. L. Ng, Z. Phys. C55, 145 (1992).

[16] L. G. Cabral-Rosetti, J. Bernabéu, J. Vidal, A. Zepeda, Eur. Phys. J. C12, 633 (2000).

[17] D. Degrassi, A. Sirlin, and W.J.Marciano, Phys. Rev. D39, 287 (1989).

[18] J. Papavassiliou, Phys. Rev. D41, 3179 (1990).

[19] J.M. Cornwall, in Proceedings of the French-American Seminar on Theoretical Aspects of Quantum Chromodynamics, Marseille, France, 1981, edited J.W. Dash (Centre de Physique Théorique, Marseille, 1982).

[20] J.M. Cornwall, Phys. Rev. D26, 1453 (1982).

[21] J.M. Cornwall and J. Papavassiliou, Phys. Rev. D40, 3474 (1989).

[22] G. Degrassi and A. Sirlin, Phys. Rev. D46, 3104 (1992);

[23] J. Papavassiliou and A. Pilaftsis, Phys. Rev. D54, 5315 (1996). 
[24] J. Papavassiliou, E. de Rafael, and N.J. Watson, Nucl. Phys. B503, 79 (1997).

[25] J. Papavassiliou, Phys. Rev. Lett. 84, 2782 (2000); Phys. Rev. D62:045006 (2000) .

[26] G. 't Hooft, Nucl. Phys. B33, 173 (1971); J.M. Cornwall and G. Tiktopoulos, Phys. Rev. D15, 2937 (1977).

[27] A. Denner, S. Dittmaier, and G. Weiglein, Phys. Lett. B333, 420 (1994); S. Hashimoto, J. Kodaira, Y. Yasui, and K. Sasaki, Phys. Rev. D50, 7066 (1994); E. de Rafael and N. J. Watson, unpublished.

[28] B.S. DeWitt, Phys. Rev. 162, 1195 (1967); G. 't Hooft, in Acta Universitatis Wratislvensis no. 38, 12th Winter School of Theoretical Physics in Kapacz, Functional and probabilistic methods in quantum field theory vol. I (1975); B.S. DeWitt, A gauge invariant effective action, in Quantum gravity II, ed. C. Isham, R. Penrose, and D. Sciama (Oxford press, 1981).

[29] L.F. Abbott, Nucl. Phys. B185, 189 (1981), and references therein.

[30] L.F. Abbott, M.T. Grisaru, and R.K. Schaeffer, Nucl. Phys. B229, 372 (1983).

[31] For some recent literature on various formal issues of the BFM see P.A. Grassi, Nucl. Phys. B462, 524 (1996); Nucl. Phys. B537, 527 (1999); Nucl. Phys. B560, 301 (1999).

[32] J. Papavassiliou, Phys. Rev D 51, 856 (1995).

[33] C. Becchi, A. Rouet, and R. Stora, Ann. Phys. (NY) 98, 287 (1976).

[34] R. C. Allen, et all., Phys. Rev. D43, R1 (1991).

[35] A. M. Mourõ, J. Pulido and J. P. Ralston, Phys. Lett. B285, 364 (1992).

[36] P. Salati, Ast. Phys. 2, 269 (1994).

[37] P. Vilain, et all., Phys. Lett. B345, 115 (1995).

[38] See also N.J. Watson, Nucl. Phys. B494, 388 (1997).

[39] For some explicit calculations demonstrating the PT mechanism of GFP cancellations in the electroweak sector of the SM see also J. Papavassiliou, Phys. Rev D 50, 5958 (1994); J. Papavassiliou and A. Pilaftsis, Phys. Rev. D58:053002 (1998).

[40] A. Denner, S. Dittmaier, and G. Weiglein, Nucl. Phys. B440, 95 (1995).

[41] A. Pilaftsis, Nucl. Phys. B487, 467 (1997)

[42] L. L. Foldy, Phys. Rev. 83, 688 (1951); Rev. Mod. Phys. 30, 471 (1958) . 